TRANSACTIONS OF THE

AMERICAN MATHEMATICAL SOCIETY

Volume 355, Number 11, Pages 4267-4296

S 0002-9947(03)03264-1

Article electronically published on July 8, 2003

\title{
ON MODEL COMPLETE DIFFERENTIAL FIELDS
}

\author{
E. HRUSHOVSKI AND M. ITAI
}

\begin{abstract}
We develop a geometric approach to definable sets in differentially closed fields, with emphasis on the question of orthogonality to a given strongly minimal set. Equivalently, within a family of ordinary differential equations, we consider those equations that can be transformed, by differential-algebraic transformations, so as to yield solutions of a given fixed first-order ODE $X$. We show that this sub-family is usually definable (in particular if $X$ lives on a curve of positive genus). As a corollary, we show the existence of many model-complete, superstable theories of differential fields.
\end{abstract}

\section{INTRODUCTION}

A classical theorem of Macintyre ([16], cf. also [4]) states that every infinite $\omega$ stable field is algebraically closed. This has led to speculation that, by analogy, every infinite $\omega$-stable differential field is differentially closed. We show here ( $\S 3)$ that this is not the case; there are many theories of differential fields admitting quantifier elimination, and more $\omega$-stable ones.

In this paper, all fields have characteristic 0 . All formulas will be quantifier-free formulas in the language of differential fields. A formula is strongly minimal if every quantifier-free definable subset, in any differential field extension, is finite or cofinite.

Shelah's work on superstable theories led to the understanding that in a theory of Morley rank $\omega$, such as differentially closed fields of characteristic 0 (with a single derivation), the strongly minimal sets control much of the structure of the models. (One must actually consider infinitely definable minimal types; it is not known if every such type arises from a strongly minimal set, in the differential field context.) In particular, 26] gave a general criterion for minimality of prime models in totally transcendental theories; this led to one of the early applications of stability theory to the subject, the independent proof in [27, 23], [11] of the non-minimality of the differential closure. All proved, in different ways, the existence of strongly minimal sets with trivial induced structure.

The following instance of Shelah's philosophy is relevant to this paper: an algebraically closed differential field $K$ is differentially closed iff every strongly minimal formula over $K$ has a solution in $K([19])$. Indeed, the general theory of superstability reduces to formulas that are either strongly minimal, or else are part of a definable principal homogeneous space for a connected definable simple group of finite Morley rank. Any such group is associated with a certain strongly minimal

Received by the editors August 1, 1998.

2000 Mathematics Subject Classification. Primary 03C60, 12H05.

The first author thanks Miller Institute at the University of California, Berkeley. 
set. A more specific study of the strongly minimal sets ([9]) and definable Galois groups ([19]) then shows that one is actually reduced to the strongly minimal case.

One is led (for this question and for others) to study the structure of strongly minimal sets. This can be divided into a number of aspects.

(1) The possible geometries associated to (strongly) minimal sets.

(2) For strongly minimal sets with nontrivial geometry, the structure of definable groups and definable group actions.

(3) For (strongly) minimal sets with trivial geometry:

a) Are there finitely or infinitely many algebraic points? What is the Galois group?

b) Are there finitely or infinitely many algebraic correspondences between two such strongly minimal sets? In particular, between a strongly minimal set and itself? If finitely many, can they be bounded uniformly?

(4) Families of strongly minimal sets. To what extent do the above properties vary uniformly in families?

The possible geometries (1) were fully classified in 9. Using these results, in his study of (2) in 19, Pillay showed that in a superstable differential field, every strongly minimal set with nontrivial geometry must have a solution in $K$.

In a differential field with quantifier elimination, types correspond to (certain) differential ideals; since these ideals satisfy a chain condition, every theory of differential fields with quantifier elimination is $\omega$-stable, and in particular superstable. Thus the result of [19] is applicable to such differential fields.

The remaining question is whether all strongly minimal types with a trivial geometry must be realized in a saturated model of the theory. It was conjectured in [19, correctly, that the answer is no.

Two structural properties of trivial strongly minimal sets are implicated in this question. First, (3a) a strongly minimal structure with trivial geometry can, in principle, have infinitely many algebraic points. Among those defined in differential algebra - taken with their induced structure over a canonical base of definition no such strongly minimal sets are known. However, if $D$ is a strongly minimal set defined in a model of DCF0, over an algebraically closed differential field $K$, and $D$ has infinitely many algebraic points in its induced structure, then $D$ will have (infinitely many) solutions in $K$.

Second, one must consider definable families of strongly minimal sets (4). In any theory of differential fields admitting elimination of quantifiers, given any definable family of definable sets (with strongly minimal generic element), the set of those that have a point must be definable (by a quantifier-free formula). In addition, there must be some non-orthogonality invariance. Given two non-orthogonal strongly minimal sets over $K$, with trivial geometry, if one has infinitely many solutions in $K$, so will the other.

To summarize, (1) and (2) are fairly well understood in general. (3) was solved in [7] for strongly minimal sets of order 1 . The current paper clears up (4) for order 1 sets (and for some others.) Putting together these structural results permits the construction of many theories of differential fields.

Orthogonality and definable families. Most of our results will concern firstorder differential equations over the constants. On an elementary level, such an 
equation can be written: $f(D x, x)=0$, where $f(y, x)$ is an ordinary field polynomial. The order of the equation is then said to be 1 , and the degree is the degree of $f$ in the $D x$ variable. This notion of degree depends on the choice of the variable $x$; it will not explicitly show up in our work. A more intrinsic invariant is the genus of the function field, generated by $x, D x, \ldots$ over the field of constants $k$. This is the function field of a complete nonsingular curve $C$; the equation can be rewritten in a less elementary but more canonical and informative fashion with the aid of a variable ranging over $C$.

We show in $\S 2$ that when the genus of $C$ is 1 or more, orthogonality to the strongly minimal set defined by such a differential equation is always a definable property. It is curious that although our subject matter consists entirely of strongly minimal sets with trivial geometries, the proof uses the Manin maps on Abelian varieties.

Strictly minimal sets on curves. In order to apply the results of $\S 2$, we need to construct strictly minimal sets living on curves of higher genus. We point out an easy, but pleasing and quite useful, correspondence between strictly minimal sets of order 1 , over the constants, and curves with 1-forms on them. To each curve over $k$ and 1-form on it, we associate a strongly minimal set. A rational map between curves and 1-forms gives rise to a definable map of strongly minimal sets. We show, in a strong form, that, conversely, every Kolchin-closed definable map between such strongly minimal sets arises in this way. In particular, strict minimality corresponds to a definite property of the 1 -forms. In genus $>1$, strict minimality implies trivial geometry. Non-orthogonality then corresponds to isomorphism of the corresponding geometric structures (curve and 1-form). A reasonably complete picture of order 1 strongly minimal sets over the constants is achieved.

Thanks to Zoé Chatzidakis, Dave Marker, Wai Yang Pong, Thomas Scanlon, Carol Wood, and the referee for remarks improving this paper.

\section{Differential and algebraic varieties}

This section is devoted to setting up the framework, and recalling and systematizing observations from [9].

$k$ will denote the field of constants in a universal domain for differential fields of characteristic $0 . k_{0}$ will be an algebraically closed subfield of $k$, and $K$ will be a differential field extension of $k_{0}$. When we consider differential equations with constant coefficients, we are thinking of $k$ as a base set rather than a definable set. It is probably necessary to have two distinct notations for these two aspects of the constants; for instance, if $p$ is a strongly minimal set, the orthogonality of $p$ to the set of constants (in the sense of Shelah) is a stronger condition than the orthogonality of $p$ to the definable set $D x=0$. In this paper, the first condition will never be considered. We will mostly use the notation $k$ when we think of it as a definable set.

A subset $X$ of an algebraic variety $V$ over the universal domain is called Zariskiclosed (respectively Kolchin-closed) if it is (locally, on each piece of an affine chart) defined by the vanishing of polynomials (respectively, differential polynomials). $X$ is called irreducible if it is not the union of two proper Zariski- (respectively, Kolchin-) closed sets. (A theorem of Kolchin states that a Zariski-irreducible Zariski-closed set is also Kolchin-irreducible.) If $X$ is an irreducible Kolchin-closed set of finite 
differential order, defined over $K$, let $K(X)$ be the field of differential rational functions on $X$. Thus $K(X)=K(a, D a, \ldots)$ if $a$ is a generic point of $X$. The differential order of $X$ is defined to be the transcendence degree of $K(X)$ over $K$. If this order is finite, then $K(X)$ is a finitely generated field extension of $K$; it is then the function field of a $K$-variety $V$, of dimension equal to the order of $X$; we then say that $X$ lives on $V$.

Observe that if we blow up a point of $V \backslash X$, then $X$ also lives on the blow-up; thus $V$ is determined by $X$ only up to birational changes.

If $X$ lives on $V$, then for generic $a \in X, D a$ belongs to the function field $K(V)$ of $V$; i.e., $D a=s(a)$ for a rational function $s$ on $V$. Thus if we are willing to remove a proper Zariski-closed subset of $V$ (where $s$ is not defined) and a proper Kolchinclosed subset of $X$ (where the equality $D x=s(x)$ fails), we obtain a representation of the form

$$
X=\Xi(V, s)=\operatorname{def}\{v \in V: D v=s(v)\} .
$$

We can take $V$ to be a smooth variety, and $s$ regular.

We will see in a moment that once $X$ is given, $V$ and $s$ are determined up to birational isomorphism, and $s$ is determined as a rational function on $V$; the target variety of $s$ will be described later. Thus we may write $\chi(X)=(V, s)$. We will see below that $\chi$ is a functor on Kolchin closed sets of finite order, yielding a close relation between them and algebraic varieties with vector fields.

The shifted tangent bundle. First, however, let us describe the target of $s$; compare [3, 2], 9]. Let $(K, D)$ be a differential field, and $V$ a variety over $K$ (possibly reducible; it may be assumed $K$-irreducible.) Consider subvarieties $V$ of affine space $\mathbb{A}^{n}$. Let $T_{D} V$ be the smallest Zariski-closed subvariety of $\mathbb{A}^{2 n}$ containing each point $\left(a_{1}, \ldots, a_{n}, D a_{1}, \ldots, D_{a_{n}}\right)$, where $\left(a_{1}, \ldots, a_{n}\right) \in V(L), L$ some differential field extending $K$.

Thus by definition, the derivation $D$ of a differential field extension $L$ of $K$ induces a map $V(L) \rightarrow T_{D} V(L)$; this map will also be denoted $D$.

(When $V$ is smooth, defined by an ideal $I, T_{D} V$ is the zero locus of $I \cup\left\{\sum \frac{\partial f}{\partial x_{i}} y_{i}+\right.$ $D f: f \in I\}$. Here $D f$ denotes the polynomial obtained from $f$ by differentiating the coefficients. The smooth case is the only one we will require.) Further, let $\pi_{V}: T_{D} V \rightarrow V$ be the obvious projection. Then it is easy to see that $\left(T_{D}(V), \pi_{V}\right)$ is functorial (so that we have $T_{D}(f): T_{D}(V) \rightarrow T\left(V^{\prime}\right)$ associated to a morphism $\left.f: V \rightarrow V^{\prime}\right)$ and that $(\#)$

$$
T_{D}(U)=\pi_{V}^{-1}(U) \subset T_{D}(X)
$$

when $U$ is an open subset of $X$. We have, for any $a \in V,(a, D a) \in T_{D} V$. Conversely, if $a \in V$ is generic over $K$, and $b \in \pi_{V}^{-1}(a)$, then there exists a derivation $D^{\prime}$ of $K(a)$ extending $D$, with $D^{\prime}(a)=b$. (Observe, directly from the definition, that if $V=V_{1} \cup V_{2}$, a union of two proper closed subsets, then $T_{D} V=T_{D} V_{1} \cup T_{D} V_{2}$. If $a \in V$ is generic over $K$, then $a \in V_{1} \backslash V_{2}$ (or $a \in V_{2} \backslash V_{1}$ ), and so $\pi_{V}^{-1}(a) \subset \pi_{V}^{-1}\left(V \backslash V_{2}\right) \subset T_{D} V_{1}$. This reduces the previous statement to the irreducible case. In this case we define a derivation $K[a] \rightarrow K[a, b]$ by $f(a) \mapsto \sum \frac{\partial f}{\partial x_{i}} b_{i}+D f(a)$, and verify trivially that it is a derivation extending $D$.)

Using $(\#)$, one glues to get a functor on arbitrary varieties, with the same properties. 
Proposition 1.1. Let $V$ be a variety, $s$ a regular section $V \rightarrow T_{D} V$. Let $X=$ $\Xi(V, s)=\{v \in V: D v=s(v)\}$.

(1) $X$ is Zariski-dense in $V$.

(2) Any Kolchin-closed $X^{\prime} \subset X$ must have the form

$$
X^{\prime}=V^{\prime} \cap X=\Xi\left(V^{\prime}, s \mid V^{\prime}\right)=\left\{v \in V^{\prime}: D v=s(v)\right\}
$$

with $V^{\prime}$ a Zariski-closed subset of $V$.

(3) If $V$ is Zariski-irreducible, then $X$ is Kolchin-irreducible.

(4) A Zariski-closed subset $V^{\prime}$ of $V$ is called s-integral if $X \cap V^{\prime}$ is Zariski-dense in $V^{\prime}$; equivalently iff $s\left(V^{\prime}\right) \subset T_{D}\left(V^{\prime}\right)$. If $V^{\prime}, V^{\prime \prime}$ are $X$-integral, then so is $V^{\prime} \cap V^{\prime \prime}$.

(5) If $V$ is smooth, or more generally if the dimension theorem holds in $V$, then it holds for $X$ : whenever $X^{\prime}, X^{\prime \prime}$ are Kolchin-closed, irreducible subsets of $X$, and $\emptyset \neq W$ a component of $X^{\prime} \cap X^{\prime \prime}$,

$$
\operatorname{ord}(W) \geq \operatorname{ord}\left(X^{\prime}\right)+\operatorname{ord}\left(X^{\prime \prime}\right)-\operatorname{ord}(X) .
$$

Proof. (1) Let $L$ be a model of DCF0. Let $a$ be a generic point of $V$, over $L . s$ is a section, so $s(a) \in \pi_{V}^{-1}(a)$. Define a derivation of the field extension $L(a)$ of $L$, extending the given derivation of $L$, and with $D a=s(a)$. Let $V^{\prime}$ be a proper subvariety of $V$, defined over $L$. Then in $L(a), a \in X \backslash V^{\prime}$. Since $L$ is existentially complete, $X \backslash V^{\prime} \neq \emptyset$ in $L$.

(2) $X^{\prime}$ is defined by the vanishing of some differential polynomials $H(x, D x, \ldots)$. Replacing $D x$ by $s(x)$ in these, we obtain regular algebraic functions $H^{\prime}(x)=$ $H(x, s(x), \ldots)$; and $H, H^{\prime}$ agree on $X$. Let $V^{\prime}$ be the Zariski-closed subset of $V$ defined by the vanishing of the $H^{\prime}$. Then $X^{\prime}=X \cap V^{\prime}$.

(3) If $X=X_{1} \cup X_{2}$, with $X_{i}$ Kolchin-closed, we have by (2) $X_{i}=X \cap V_{i}$, with $V_{i}$ Zariski-closed; by (1), $V_{1} \cup V_{2}=V$; by irreducibility, some $V_{i}=V$, so $X_{i}=X$.

(4) This reduces to the case when $V$ is affine. There it is equivalent to a classical lemma of Kolchin's ([1]), to the effect that the prime components of a differential ideal are also differential ideals.

(5) By (2), (4) we have $X^{\prime}=V^{\prime} \cap X, X^{\prime \prime}=V^{\prime \prime} \cap X$; and $\operatorname{ord}\left(X^{\prime}\right)=\operatorname{dim}\left(V^{\prime}\right)$, $\operatorname{ord}\left(X^{\prime \prime}\right)=\operatorname{dim}\left(V^{\prime \prime}\right), \operatorname{ord}(X)=\operatorname{dim}(V)$. If $V_{1}, \ldots, V_{m}$ are the components of $V^{\prime} \cap V^{\prime \prime}$, letting $W_{i}=V_{i} \cap X$, we have $V^{\prime} \cap V^{\prime \prime}=\bigcup W_{i}$. Thus $W=W_{i}$ for some $i$. It follows that $\operatorname{ord}(W)=\operatorname{dim}\left(V_{i}\right) \geq \operatorname{dim}\left(V^{\prime}\right)+\operatorname{dim}\left(V^{\prime \prime}\right)-\operatorname{dim}(V)$.

Fix a "universal domain" $\mathbb{U}$; it is a model of DCF0. Varieties and Kolchin-closed sets are taken to be over $\mathbb{U}$.

If $K$ is a differential subfield of $\mathbb{U}$, let $\mathcal{C}_{\mathrm{Kol}}(K)$ be the category of Kolchin-closed sets $V$ defined over $K$ of finite differential order, up to subsets of smaller order; thus in $\mathcal{C} 1 V$ and $V \backslash V^{\prime}$ are identified if $\operatorname{ord}\left(V^{\prime}\right)<\operatorname{ord}(V)$. A morphism $V \rightarrow U$ is a differential regular map $g:\left(V \backslash V^{\prime}\right) \rightarrow U$, defined over $K$, for some $V^{\prime} \subset V$ of smaller order than $V \cdot \mathcal{C}_{\mathrm{Kol}}(\mathbb{U})$ is denoted simply $\mathcal{C}_{\mathrm{Kol}}$.

Let $\mathcal{C}_{Z}$ be the category of pairs $(V, s), V$ an algebraic variety, $s: V \rightarrow T_{D} V$ a regular section, up to subsets of smaller dimension: $(V, s)$ is identified with $U, s \mid U$ if $U$ is a dense open subset of $V$. A morphism $(V, s) \rightarrow(U, t)$ is a regular map $h:\left(V \backslash V^{\prime}\right) \rightarrow U$ such that $t \circ h=T_{D}(h) \circ s$, for some $V^{\prime} \subset V$ of smaller dimension than $V \cdot \mathcal{C}_{Z}(K)$ is the subcategory whose objects and morphisms are to be defined over $K$. 
Remark 1.1. Let $\mathcal{C}_{\mathrm{Kol}} \#$ be the finer category of Kolchin-closed sets of finite differential order, and differential regular maps between them. Thus $\mathcal{C}_{\text {Kol }}$ is a quotient of

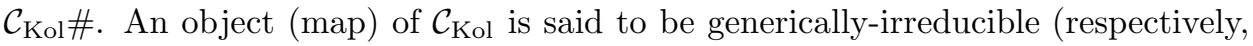
dominant) if it is represented by an irreducible object (respectively, by a surjective

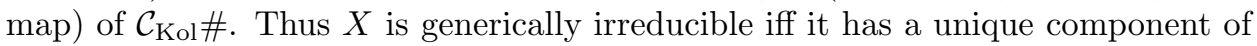
maximal dimension, or iff it has a unique type of elements of maximal order.

The questions of orthogonality studied in this paper relate to $\mathcal{C}_{\text {Kol }}$, rather than

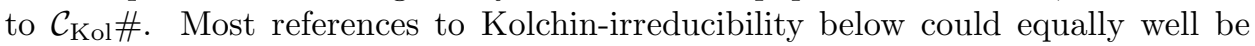
replaced by generic irreducibility, and the difficulties associated to Kolchin-irreducibility are irrelevant. Nevertheless we give a brief discussion of these issues in 33.1

Proposition 1.2. The functor $\Xi$ induces an equivalence of categories $\mathcal{C}_{\mathrm{Kol}} \rightarrow \mathcal{C}_{Z}$, with inverse $\chi$. The equivalence translates differential order to dimension, respects fields of definition, and is compatible with "image" and "generic fiber": $f: X_{1} \rightarrow$ $X_{2}$ is dominant iff $\chi(f)$ is, and in this case, if $Y$ is a generic fiber of $f$, then $\chi(Y)$ is a generic fiber of $\chi(f)$.

In particular, if $1 \leq m \in \mathbb{N}$ and $f$ is generically $m$-to- 1 , then so is $\chi(f)$.

Proof. Fix a differential field $K \subset \mathbb{U}$; let us show the equivalence between the objects and morphisms of $\mathcal{C}_{\mathrm{Kol}}(K), \mathcal{C}_{Z}(K)$.

First we describe $\Xi$. Given an object $(V, s)$ of $\mathcal{C}_{Z}(K)$, let $\Xi(V, s)={ }_{\text {def }}\{v \in V$ : $D v=s(v)\}$. It was shown in 1.1 that the order of $\Xi(V, s)$ equals the dimension of $V$. In particular, if $V^{\prime}$ is a subvariety of $V$ of smaller dimension, $U=V \backslash V^{\prime}$, then $\Xi(V, s) \backslash \Xi(U, s)$ has smaller order than $\Xi(V, s)$; so $\Xi$ is well-defined on the objects of $\mathcal{C}_{Z}$, into those of $\mathcal{C}_{\mathrm{Kol}}$.

Now if $f:(V, s) \rightarrow(U, t)$ is a morphism in $\mathcal{C}_{Z}(K)$, define $\Xi(f)=f \mid \Xi(V, s)$.

With the intervention of the category of finitely generated $K$-algebras, endowed with a derivation extending the derivation $D_{K}$ of $K$, we showed above that every object of $\mathcal{C}_{\mathrm{Kol}}(K)$ is in the image of $\Xi\left(\mathcal{C}_{Z}(K)\right)$; and similarly so is every morphism.

It remains to show that $\Xi(f)$ is an isomorphism in $\mathcal{C}_{\mathrm{Kol}}$ iff $f$ is an isomorphism in $\mathcal{C}_{Z}$. Note that $f$ is an isomorphism in these categories iff $f$ is dominant, and generically 1-1. Thus it suffices to prove the statement concerning preservation of dominance, and of generic fibers.

Let $f:(V, s) \rightarrow(U, t)$. Assume $f: V \rightarrow U$ is dominant. Let $a \in \Xi(U, t)$ be a generic point over $K$. Let $V_{a}=f^{-1}(a)$. Then $V_{a}$ is a nonempty $K(a)$ variety. Moreover, $T_{D}(f): T_{D}(V) \rightarrow T_{D}(U),(a, D a) \in T_{D}(U)$, and $T_{D}\left(V_{a}\right)=$ $T_{D}(f)^{-1}(a, D a)$. From $t \circ f=T_{D}(f) \circ s$ it follows that $s_{a}=s \mid V_{a}$ is a section of $T_{D}\left(V_{a}\right)$. Thus by 1.1, $\Xi\left(V_{a}, s_{a}\right)$ is Zariski-dense in $V_{a}$. In particular, it is non-empty; this shows that $\Xi(f)$ is dominant. Moreover, $a$ is a generic point of $U$; so $V_{a}$ is a generic fiber of $f$; this proves the preservation of generic fibers. In particular, since $\Xi\left(V_{a}, s_{a}\right)$ is Zariski-dense in $V_{a}, V_{a}$ is finite iff $\Xi\left(V_{a}, s_{a}\right)$ is finite, and in this case they are equal.

See 21] for the notion of elimination of imaginaries, and the proof that it holds in the theory DCF0 of differential fields of characteristic 0 (Theorem 7 there). Actually we only require codes for finite sets given by symmetric functions in any field.

Lemma 1.2. Let $X$ be an irreducible Kolchin-closed set living on a variety $V$. Let $E$ be a definable equivalence relation on $X$ with finite classes. Then there exist a variety $U$, a rational map $f: V \rightarrow U$ and a Kolchin-closed $Y$ living on $U$, such 
that, for all $a \in X$ outside a proper Kolchin-closed subset, $f(a) \in Y$ and $f^{-1}(f(a))$ is the $E$-class of a. If $L_{0}$ is a differential field over which $X, E$ (and $V$ ) are defined, one may take $Y, U, f$ defined over $L_{0}$.

Proof. Using elimination of imaginaries in DCF, let $f_{0}: X \rightarrow Y$ be a definable map whose fibers are the classes of $E$. Removing a Zariski-closed proper subset of $X$, we may assume $Y$ is a Kolchin-closed irreducible set, and $f_{0}$ is a dominant regular differential rational map. $Y$ lives on a variety $U$, and by Proposition $1.2 f_{0}$ is induced from a unique $f: V \rightarrow U$. Say $f_{0}^{-1}(b)$ has $m$ points for generic $b \in Y$; then by 1.2, $f^{-1}(u)$ has $m$ points for generic $u \in U$; removing a proper Zariskiclosed subset of $U$ (that necessarily intersects $Y$ in a proper Kolchin-closed subset of $Y$, since $Y$ is Zariski-dense in $U$ ), we may assume $f^{-1}(u)$ has at most $m$ points for any $u \in U$. It follows that $f^{-1}(b)=f_{0}^{-1}(b)$ for generic $b \in Y$, as promised. It is clear that $Y, f_{0}$ may be taken defined over $L_{0}$; by functoriality so are $U, f$.

Remark 1.3. We will mostly work in this paper with Kolchin-closed sets defined over $k_{0} \subset k$. In this case $T_{D} V=T V$ is the tangent bundle (for smooth $V$ defined over $\left.k_{0}\right)$.

\section{Strictly minimal SeTS ON CURVES}

We study non-orthogonality classes of degree one strongly minimal sets, defined over the constants. In genus $\geq 2$, we show that such classes are in 1-1 correspondence with pairs $(C, \omega), C$ a curve of genus $\geq 2$ defined over $k, \omega$ a rational 1-form on $C$, defined over $k$, that is not the pullback of any other 1-form on a curve of smaller genus.

Using this geometric representation of non-orthogonality classes, we will construct a class of strongly minimal sets, with trivial induced structure, living on curves of genus $\geq 2$ over the constants.

In $\S \S 2.1$ and 2.2 , we use similar methods to study strongly minimal sets living on $\mathbb{P}^{1}$ or on elliptic curves.

Strongly minimal sets defined over generic parameters can be analyzed by very similar methods; but the situation over non-generic parameters remains to be described. Generalizations to higher dimensions also remain open and of great interest.

Algebraic curves. The material on curves that we will require can be found in the first chapter of Hartshorne's book [5], or in any basic text, e.g. [15]. We briefly summarize it. Our curves will be nonsingular and complete; they will be defined over an algebraically closed field (of characteristic 0 ). A rational morphism between such curves is automatically regular (cf. the corollary to Theorem VI.1.1 in [15]). Associated to a curve $C$ is the space of rational 1-forms on $C$, i.e., rational maps $\omega$ on $C$ such that $\omega(c)$ is an element of the dual to the tangent space $T_{c} C$ to $C$ at $c$. If $0 \neq \omega$ is one such form, then any other equals $f \omega$ for some rational function $f$ on $C$. If $\omega$ is nonzero, it will have a finite number $z$ of zeroes and a finite number $p$ of poles, and $z-p=2 g-2$, where $g$ is a number depending on $C$ alone, called the genus of the curve. If $c$ is a simple pole of $\omega$, then the residue of $\omega$ at $c$ is well-defined; the sum of residues is 0 . Forms with no poles are called global. The set of global 1-forms on $C$ forms a vector space of dimension $g$, denoted $\Omega^{1}(C)$. (If $C$ is defined over $L$, then the global 1 -forms defined over $L$ form a $g$-dimensional $L$-space.) The only curve of genus 0 is the projective line. On it there are no global 
1-forms, since $2 g-2=-2<0$. Curves $C$ of genus $g \geq 1$, with a distinguished point 0 , admit an embedding into an algebraic group $J$ of dimension $g \geq 1$, the Jacobian; it is an isomorphism iff the genus is 1 . For any element $T$ of $\left(T_{0} J\right)^{*}$ there exists a translation-invariant 1-form $\omega$ with $\omega(0)=T$; if $T \neq 0$ then this $\omega$ has no zeroes or poles; it restricts to a global 1-form on $C$, and all global 1-forms on $C$ are obtained in this way, as one can check by comparing dimensions.

In this section, $C$ will denote a smooth curve defined over a field $K . K(C)$ denotes the field of rational functions on $C$ defined over $K$. There is a 1-1 correspdence between non-zero 1-forms $\omega$, and non-zero vector fields $t$ on $C$, given by the relation $\omega(p) t(p)=1$ for almost all $p$. This relates the 1-forms used here to the vector fields of the previous section. We will work with curves defined over the constants, so the shifted bundle will not arise.

The pullback of a 1 -form $\omega$ by a regular map $f: C \rightarrow C^{\prime}$ is defined as $f^{*} \omega=\omega \circ d f$, where $\omega$ is viewed as a map on $T C^{\prime}$, and $d f: T C \rightarrow T C^{\prime}$.

Let $C$ be a nonsingular curve, defined over a field $k_{0}$ contained in the constants. If $\omega$ is a nonzero rational 1 -form on $C$, i.e. a rational section of the cotangent bundle, let

$$
\Xi(C, \omega)=\Xi\left(C^{\prime}, t\right),
$$

where $Z$ is the finite set of zeroes of $\omega, C^{\prime}=C \backslash Z$, and $t$ is the vector field on $C^{\prime}$ satisfying $t \omega=1$. In other words, $\Xi(C, \omega)=\{a \in C: \omega(a) D a=1\}$, and this is taken to include a pole $a$ of $\omega$ iff $D a=0$.

Note that $\Xi(C, \omega)$ is Kolchin-closed: the equations $D x=t(x)$ and $\omega(x) D x=1$ define $\Xi(C, \omega)$ away from the zeroes (respectively, the poles) of $\omega$; between them they show that $C$ is closed near every point, hence closed. Moreover since $\Xi(C, \omega) \subset$ $C^{\prime}$, by 1.1, $\Xi(C, \omega)$ is Kolchin-irreducible.

(Therefore the treatment of zeroes and poles is forced by the requirement that $\Xi(C, \omega)$ be closed and irreducible.)

Moreover, by [1.1, all infinite definable subsets of $\Xi\left(C^{\prime}, t\right)$ have the form $\Xi\left(C^{\prime}, s\right) \cap$ $C^{\prime \prime}$ for a constructible $C^{\prime \prime} \subset C^{\prime}$; since $C$ is a curve, $C^{\prime \prime}$ is finite or cofinite, so $\Xi(C, s)$ is strongly minimal.

Remark 2.1. Observe that if $\omega$ is defined over $k$, the finitely many poles of $\omega$ are also defined over $k$; hence $D a=0$ automatically at the poles. Thus the points of $\Xi(C, \omega)$ algebraic over the base of definition are precisely the poles of $\omega$.

Remark 2.2. Beyond dimension 1, 1-forms and vector fields play different roles; over the constants, 1-forms are associated with definable sets of co-order 1 (cf. [7]). They present an advantage if one is interested in strongly minimal sets defined over generic parameters. Here our choice of 1 -form over vector field notation is somewhat arbitrary.

Lemma 2.3. Let $Y$ be any strongly minimal set, defined over the constants, living on $C$. Then, up to a finite set, either $Y=C(k)$ (so $Y$ is defined by $D x=0$ ) or $Y=\Xi(C, s)$ for some rational 1-form s defined over the constants.

Proof. Since $Y$ lives on $C$, in local coordinates on a Zariski-open affine, $D y=h(y)$ for $y \in Y$, where $h$ is a rational section of the tangent bundle of $C$. If $h \neq 0$, then we can define a 1 -form $s$ by $s(y) h(y)=1$. Then $\Xi(C, s)$ coincides with $Y$, up to a finite set. 
A strongly minimal set $\Xi(C, \omega)$, with $\omega \in \Omega^{1}(C)$, will be called global. Note that they form a definable family of strongly minimal sets.

A 1 -form $s$ on $C$ will be called essential if there is no rational map $g: C \rightarrow C^{\prime}$ of degree $d>1$, and a rational 1-form $s^{\prime}$ on $C^{\prime}$, with $s=g^{*} s^{\prime}$.

It will be shown later that in genus $\geq 2$, generic global 1-forms are essential. $\left(\Omega^{1}(C)\right.$ has a basis $s_{1}, \ldots, s_{\gamma}$ of global 1 -forms defined over $k_{0}$. Let $t=\left(t_{1}, \ldots, t_{\gamma}\right)$ be a generic (over $\left.k_{0}\right) \gamma$-tuple of constants, and let $s=\sum t_{i} s_{i}$. This is a generic element of $\Omega^{1}(C)$ (defined over $k_{1}=k_{0}(t)$ ).)

Proposition 2.1. Let $C$ be a complete nonsingular curve over $k$, of genus $\gamma>1$. Let $s$ be an essential global 1-form on $C$, defined over $k$. Then $\Xi(C, s)$ is strictly minimal, with trivial induced structure.

Two such sets $\Xi\left(C_{1}, s_{1}\right), \Xi\left(C_{2}, s_{2}\right)$ are not orthogonal if and only if there exists an isomorphism $g: C_{1} \rightarrow C_{2}$ with $s_{1}=g^{*} s_{2}$.

We insert some lemmas prior to the proof of the proposition. The curves occuring in the proposition and the lemmas are assumed to be defined over $k$; the 1-forms and rational maps, not always.

Lemma 2.4. Let $g: C_{1} \rightarrow C_{2}$ be a dominant regular map between nonsingular curves, and $s_{2}$ a 1-form on $C_{2}$, all defined over $k$. Let $s_{1}=g^{*} s_{2}$ be the pullback by $g$ to $C_{1}$. Then $g^{-1} \Xi\left(C_{2}, s_{2}\right)=\Xi\left(C_{1}, s_{1}\right)$.

Proof. Let $c_{1} \in C_{1}, c_{2}=g\left(c_{1}\right)$. Then

$$
s_{1}\left(c_{1}\right) D c_{1}=s_{2}\left(c_{2}\right)(d g)\left(D c_{1}\right)=s_{2}\left(c_{2}\right) D\left(g\left(c_{1}\right)\right)=s_{2}\left(c_{2}\right) D c_{2} .
$$

Thus $s_{1}\left(c_{1}\right) D c_{1}=1$ iff $s_{2}\left(c_{2}\right) D c_{2}=1$.

Lemma 2.5. Let $g \in k(C) \backslash k$. Let $s$ be a 1-form on $C$. Then $g(\Xi(C, s)) \backslash k$ is infinite.

Proof. We may write $d g=h s$ for some rational function $h$ on $C . g, s, h$ are regular on a cofinite $C^{\prime} \subset C$. If $h=0$ then $g$ is constant, $g \in k$. Otherwise, for $c \in \Xi\left(C^{\prime}, s\right)$, $D g(c)=(d g)_{c} D c=h(c) s(c) D c=h(c)$. Thus if $g \Xi(C, s)$ is almost contained in the constants, then $h$ vanishes on $\Xi\left(C^{\prime}, s\right)$; hence $h=0$.

It follows from these lemmas that the image under a rational map of $\Xi\left(C_{1}, s_{1}\right)$, if it lives on a curve $C_{2}$, must (a.e.) have the form $\Xi\left(C_{2}, s_{2}\right)$ for some 1-form $s_{2}$.

Lemma 2.6. If $\Xi(C, s)$ and $\Xi\left(C, s^{\prime}\right)$ have infinite intersection, then $s=s^{\prime}$.

Proof. $C(k), \Xi(C, s)$, and $\Xi\left(C, s^{\prime}\right)$ are all strongly minimal. By Lemma2.5 $\Xi(C, s) \cap$ $C(k)$ is finite. So $D c \neq 0$ for $c \in \Xi(C, s) \cup \Xi\left(C, s^{\prime}\right)$ (excepting perhaps finitely many points). Thus for infinitely many points $c$ in the intersection $\Xi(C, s) \cap \Xi\left(C, s^{\prime}\right)$ we have $s(c) D c=1=s^{\prime}(c) D c$, and $D c \neq 0$. Thus $s(c)=s^{\prime}(c)$ for infinitely many $c$, so $s=s^{\prime}$.

Lemma 2.7. Let $g: C_{1} \rightarrow C_{2}$ be a dominant rational map between nonsingular curves, all defined over $k$. Let $s_{i}$ be a 1-form on $C_{i}$. Assume $g \Xi\left(C_{1}, s_{1}\right) \subset \Xi\left(C_{2}, s_{2}\right)$ (perhaps up to a finite set). Then $s_{1}=g^{*} s_{2}$.

Proof. By Lemma 2.4, $g^{-1} \Xi\left(C_{2}, s_{2}\right)=\Xi\left(C_{1}, g^{*} s_{2}\right)$ (a.e.). Thus the intersection of $\Xi\left(C_{1}, s_{1}\right)$ with $\Xi\left(C_{1}, g^{*} s_{2}\right)$ is infinite. By [2.6] $s_{1}=g^{*} s_{2}$. 
Lemma 2.8. Let $C$ be a complete nonsingular curve, $s$ a global 1-form on $C$, both defined over $k$. Then there is no rational function $g \in k(C)$ such that $g \Xi(C, s)$ lives on $\mathbb{P}^{1}$.

More generally, if $g: C \rightarrow C^{\prime}$ is a rational function into a complete nonsingular curve, and $g \Xi(C, s)=\Xi\left(C^{\prime}, s^{\prime}\right)$, then $s^{\prime}$ is a global 1-form on $C^{\prime}$.

(The referee kindly provided a reference: I.R. Shafarevich, Basic Alebraic Geometry, 2nd ed., Springer-Verlag, Berlin-Heidelberg 1994, Theorem 6.1.2.)

Proof. As $C$ is nonsingular and $C^{\prime}$ is complete, $g: C \rightarrow C^{\prime}$ is a morphism; it is surjective since $C$ is complete.

By $2.7 s=g^{*} s^{\prime}=(d g) \circ\left(s^{\prime} \circ g\right)$. We will show that if $s$ is regular at $p \in C$, then $s^{\prime}$ is regular at $p^{\prime}=g(p) \in C^{\prime}$. Trivialize the tangent bundles of $C$ and of $C^{\prime}$ near $p$ and $p^{\prime}$, and let $t, t^{\prime}$ be rational functions on $C, C^{\prime}$, viewed as sections of the trivialized bundles, such that $s t=1, s^{\prime} t^{\prime}=1$. We have to show that if $t^{\prime}\left(p^{\prime}\right)=0$, then $t(p)=0$. Now

$$
g^{\prime}(x) \cdot t(x)=\left(t^{\prime}(g(x)) .\right.
$$

Choosing a parameter for $C$ at $p$, we see that if $g^{\prime}$ vanishes to order $m$ at $p$, then $g(p)=p^{\prime}$ to order $m+1$; since $t^{\prime}$ vanishes to some order $k \geq 1$ at $p^{\prime}, t^{\prime}(g(x))$ vanishes to order $(m+1) k>m$ at $x=p$; so $t(x)$ must vanish to order $(m+1) k-m>0$.

In the case of $\mathbb{P}^{1}$, all nonzero rational 1 -forms have at least 2 poles (poles zeroes $=2 g-2$ ), so the first assertion follows.

Lemma 2.9. Let $s$ be an essential rational 1-form on $C$ defined over $k$. Let $E$ be a definable equivalence relation on $\Xi(C, s)$, defined over $k$, with finite classes. Then almost every class of $E$ has one element.

Proof. Suppose otherwise. By Lemma 1.2 there exist $C^{\prime}$ and $g: C \rightarrow C^{\prime}$ with $\operatorname{deg}(g)>1$ such that $g(\Xi(C, s))$ lives on $C^{\prime}$. So (up to a finite set) $g \Xi(C, s)=$ $\Xi\left(C^{\prime}, s^{\prime}\right)$ for some rational 1-form $s^{\prime}$ on $C^{\prime}$. By 2.7, $s=g^{*} s^{\prime}$. But this contradicts the assumption that $s$ is essential.

Lemma 2.10. Let $s$ be a 1-form on a curve $C$, defined over $k$. Assume $s$ is not the pullback of a form on $\mathbb{P}^{1}$ (the form and the rational function both defined over $k)$. Then $\Xi(C, s)$ is non-orthogonal to the constants iff there exist an elliptic curve $E$, a rational function $g: C \rightarrow E$, and an invariant 1-form $\omega$ on $E$, all defined over $k$, such that $s=g^{*} \omega$.

Proof. In one direction, if $s=g^{*} \omega$, then $g \Xi(C, s)=\Xi(E, \omega)$. If $e \in E(k)$, then translation by $e$ leaves $\omega$ invariant, hence leaves $\Xi(E, \omega)$ invariant. Thus $\Xi(E, \omega)$ is a coset of $E(k)$, so this set is non-orthogonal to the constants.

Assume conversely that $\Xi(C, s)$ is non-orthogonal to the constants. First suppose $\Xi(C, s)$ is not almost-orthogonal to $k$. Then there exists $c \in k, c \notin \operatorname{acl}\left(k_{0}\right)\left(=k_{0}\right)$, $c \in \operatorname{acl}\left(k_{0}(a)\right)$ for some $a \in \Xi(C, s)$. The set of conjugates of $c$ over $k_{0}(a)$ is a finite subset of $k$; by elimination of imaginaries, it is coded by a tuple $\left(t_{1}, \ldots, t_{m}\right)$ of elements of $k$; each $t_{i}$ is definable over $k_{0}(a)$, and some $t_{i}$ must be transcendental over $k_{0}$. Thus there exists a nonconstant $f \in k(C)$ with $f \Xi(C, s) \subset k$ (a.e.). However, this contradicts 2.5 .

Next, suppose $\Xi(C, s)$ is almost orthogonal, but not orthogonal, to $k$. We will use the basic results on almost-orthogonality (cf. [8], or the section on the binding group on [20]). There exists an equivalence relation $\sim$ on $\Xi(C, s)$ with finite classes, 
definable over $k_{0}$, such that $\Xi(C, s) / \sim$ is $k$-internal; there exist a set $P$ differing from $\Xi(C, s) / \sim$ by a finite set, and a definable transitive action of a connected definable group $G$ on $P$. $G$ is $k$-internal. We could use here the general theory of definable groups to pass to an algebraic group action, but instead, since we are dealing with curves, we can obtain the required information by just looking at single elements of $G$. By $1.2 \sim$ is the kernel of a rational function $g: C \rightarrow E$ ( $E$ a curve over $\left.k_{0}\right)$. By 2.6, $g \Xi(C, s)=\Xi(E, \omega)$ for some $\omega$; and $s=g^{*} \omega$. Thus we have a definable transitive action of $G$ on a set $P^{\prime}$ differing from $\Xi(E, \omega)$ by a finite set. By 1.2 each element of $g$ yields a birational map $E \rightarrow E$; since $E$ is a complete nonsingular curve, such a map corresponds to a unique biregular map $E \rightarrow E$. By [5], IV, Ex. 5.2, having infinitely many automorphisms, $E$ cannot have genus $>1$; so $E$ has genus 1 . By [5], Cor. 4.7, the group of translations of $E$ has finite index in the group of all automorphisms of $E$; so infinitely many elements of $G$ must act on $E$ by translations. For $g \in G$ we have $g \Xi(C, \omega))=\Xi(C, \omega)$ ) (up to a finite set), so $\omega=g^{*} \omega$. Thus $\omega$ is invariant under infinitely many elements of the translation group, and hence by strong minimality of $E$ under all translations.

Remark 2.11 (See also $\S 2.2$ below). It is possible to delete the hypothesis that $s$ is not a 1 -form on $\mathbb{P}^{1}$, and change the conclusion to: $s$ is the pullback of an invariant 1-form on an elliptic curve, or $G_{a}$, or $G_{m}$. This follows from the above together with [17, 6.12. It should be possible to give another proof along the above lines.

Proof of Proposition 2.1. By Remark 2.1, $\Xi(C, s)$ has no algebraic points.

By [2.9, every definable equivalence relation on $\Xi(C, s)$ with finite classes has infinitely many 1-element classes; since any exceptional classes would consist of algebraic points, all classes must have a single element.

Note that $\Xi(C, s)$ is orthogonal to the constants $k$. Otherwise, by 2.10 , there exists a rational function $g: C \rightarrow C^{\prime}, C^{\prime}$ either of genus 0 , or a 1-dimensional algebraic group, over $k$ with an invariant 1-form $\omega$ on $C^{\prime}$, and $s=g^{*} \omega$. Since $s$ is essential, $g$ must have degree 1 . But $C^{\prime}$ has genus 0 or 1 , while $C$ is assumed to have genus $>1$, a contradiction.

By [7], $\Xi(C, S)$ has $\aleph_{0}$-categorical induced structure, and trivial geometry.

It follows that $\operatorname{acl}(a)=\{a\}$ for $a \in \Xi(C, s)$. Indeed by $\aleph_{0}$-categoricity, the equivalence relation $\operatorname{acl}(x)=\operatorname{acl}(y)$ is definable, and has finite classes, so 2.9]applies, contradicting essentialness.

By the triviality of the geometry, $\operatorname{acl}\left(a_{1}, \ldots, a_{n}\right)=\left\{a_{1}, \ldots, a_{n}\right\}$ for all $a_{1}, \ldots, a_{n} \in$ $\Xi(C, s)$. By strong minimality, $\Xi(C, s)$ has no induced structure at all.

As for the orthogonality, assume $\Xi(C, s), \Xi\left(C^{\prime}, s^{\prime}\right)$ are not orthogonal. Then by triviality of the geometry they are not almost orthogonal, so there exists a $k$ definable finite-finite correspondence between them; by triviality of the algebraic closure of each, this correspondence is a bijection; so there exists a rational $g: C \rightarrow$ $C^{\prime}$ with $s=g^{*} s^{\prime}$ defined over $k$. As above, $g$ must be birational, and therefore an isomorphism.

Remark 2.12. The following purely geometric statement follows from Proposition 2.1; if $C$ is a complete nonsingular curve of genus $\geq 2, \omega$ an essential 1-form on $C$, and $C^{\prime}$ is any other curve, with a rational 1-form $\omega^{\prime}$, then there exists at most one rational map $g: C^{\prime} \rightarrow C$ with $g^{*} \omega=\omega^{\prime}$.

Conversely, this geometric fact, applied to the normalization of a correspondence on $C$, implies that there are no nontrivial correspondences on $X(C, \omega)$, and therefore 
$X(C, \omega)$ is strictly minimal. A direct geometric proof would thus circumvent the need to quote [7] in the proof of Proposition 2.1]

\section{Existence of essential 1-forms.}

Lemma 2.13. Let $C$ be a complete nonsingular curve over $k_{0}$, of genus $>1$. There exists a finite or countable union $\mathcal{E}=\bigcup_{l} S_{l}$ of proper subspaces of $\Omega^{1}(C)$, such that any 1-form in $\Omega^{1}(C) \backslash \mathcal{E}$ is essential.

There exists an essential global 1-form, defined over $k_{0}$.

Proof. Let $J$ be the Jacobian of $C$, and let $\iota: C \rightarrow J$ be an embedding. The global 1-forms of $C$ are precisely the pullbacks of invariant 1-forms on $J$.

Claim 2.14. Assume the global 1-form s on $C$ is not essential. Then there exist an Abelian variety $J^{\prime}$ with $\operatorname{dim}\left(J^{\prime}\right)<\operatorname{dim}(J)$, a homomorphism $f: J \rightarrow J^{\prime}$ of Abelian varieties, and $\omega \in \Omega^{1}\left(J^{\prime}\right)$, with $s=(f \iota)^{*} \omega$.

Proof of Claim. Since $s$ is inessential, $s=g^{*} s^{\prime}$ for some $g: C \rightarrow C^{\prime}$ with $\operatorname{deg}(g)>1$. Let $\iota^{\prime}: C^{\prime} \rightarrow J^{\prime}$ be the Jacobian of $C^{\prime}$. The dimension of $J^{\prime}$ is the genus of $C^{\prime}$; it is smaller than that of $C$ by the Hurwitz genus formula. Let $f: J \rightarrow J^{\prime}$ be the map induced by $g$, so $\iota^{\prime} g=f \iota$. Then $s^{\prime}=\iota^{\prime *} \omega$ for some $\omega$; and $s=\left(\iota^{\prime} g\right)^{*} \omega=(f \iota)^{*} \omega$.

Given an Abelian subvariety $K$ of $J$, let $\pi_{K}: J \rightarrow(J / K)$ be the canonical map. Let $V_{K}$ be the subspace of $\Omega^{1}(J)$ :

$$
V_{K}=\left\{\pi_{K}{ }^{*} \omega: \omega \in \Omega^{1}(J / K)\right\}=\left\{s \in \Omega^{1}(J): s \mid K=0\right\} .
$$

Note that $V_{K}$ is a subspace of $\Omega^{1}(J)$ of dimension $\operatorname{dim}(J / K)$; if $K$ is infinite, then $V_{K}$ is a proper subspace.

By the claim, every inessential 1-form is contained in $V_{K}$ for some infinite $K$. There are only countably many Abelian subvarieties $K$ of $J$. This proves that the inessential global forms are contained in a countable union of proper subspaces.

Note also that $V_{K} \subset V_{K^{\prime}}$ if $K^{\prime} \subset K$. Thus every inessential 1-form is contained in $V_{K}$ for some minimal proper subvariety $K$ of $J$.

For the last statement, say $C$ is defined over a finitely generated field $L \subset k_{0}$. All connected Abelian subvarieties of $J$ are defined over a fixed finite extension of $L$, obtained by adding some torsion points (cf. 6]; the statement is well-known to algebraic geometers but we do not know a reference). We may thus assume they are all defined over $L$. Thus also all $V_{K}$ are defined over $L$. But for any $\gamma$-dimensional vector space $V$ defined over $L$, and any extension field $L^{\prime}$ of $L$ with $\left[L^{\prime}: L\right]>\gamma$, $V\left(L^{\prime}\right)$ has a point avoiding all proper $L$-definable subspaces.

(This last point can be sharpened, using Remark 2.15 below.)

Remark 2.15. As a rule, $\mathcal{E}$ is a finite union of proper subspaces. The exception occurs when the Jacobian $J$ of $C$ is isogenous to a direct power $E^{\gamma}$ of an elliptic curve $E, \gamma \geq 2$. In this case, and in this case only, $\mathcal{E}$ is a countably infinite union of proper subspaces.

In the exceptional case, $\mathcal{E}$ is the union of all codimension 1 subspaces of $\Omega^{1}(E)$, whose corresponding point, in appropriate projective coordinates, is rational over the field of fractions of the ring of endomorphisms of $E$ (the complex multiplication field of $E$ ). 
Remark 2.16. Any instance of the exceptional case in 2.15 provides an example of a family of strongly minimal sets $\left\{\Xi(C, s): s \in \Omega^{1}(C)\right\}$, whose generic member is strictly minimal, orthogonal to $k$, and lives on a high genus curve; but a Kolchindense collection of special members are non-orthogonal to the constants. We will show in $\S 3$ how to construct a superstable differential field in which some generic element of this family can have no points. For exceptional families of this type, this cannot be improved to say that no generic element of the family has points (since, taking into account [19], in any superstable differential field, the strongly minimal sets non-orthogonal to $k$ must have infinitely many points).

Proof of Remark 2.15. We use here classical theorems of Severi and de Franchis; cf. [28], though a more elementary proof ought to be possible.

Consider rational maps $g: C \rightarrow C^{\prime}$ of degree $>1$, defined over $k$. Given the pair $(g, C)$, the family of pullbacks via $g$ of forms in $\Omega^{1}\left(C^{\prime}\right)$ constitutes a proper subspace $V\left(g, C^{\prime}\right)$ of $\Omega^{1}(C)$ (using the Hurwitz formula again).

Over $k$, there are only countably many curves of genus 1 dominated by $C$ (quotient elliptic curves of the Jacobian of $C$ ), and finitely many of higher genus (theorem of Severi). For each such $C^{\prime}$, there are countably many possibilities for $g$; and actually finitely many for genus $>1$, by the theorem of de Franchis.

Thus a finite union of subspaces contained in $\mathcal{E}$ accounts for all $C^{\prime}$ of genus $>1$. It remains to consider maps from $C$ into elliptic curves.

Up to isogeny, only finitely many elliptic curves are homomorphic images of the Jacobian $J$ of $C$. Fix representatives (over $k_{0}$ ) $E_{1}, \ldots, E_{r}$ of these isogeny classes. Let $K_{i}$ be the intersection of the kernels of all homomorphisms $J \rightarrow E_{i}$. Then $K_{i}$ is connected, and $J / K_{i}$ is isogenous to a power of $E_{i}$.

If each $K_{i}$ is nonzero, then in the notation of the proof of Lemma 2.13. $V_{K_{i}}$ contains $V\left(g, E^{\prime}\right)$ for each $E^{\prime}$ in the isogeny class of $E_{i}$, and each $g$. Thus if each $V_{K_{i}}$ is a proper subspace of $\Omega^{1}(C)$, we are done.

Otherwise, $K_{i}=0$ for some $i$. It follows that we are in the exceptional case: $J$ is isogenous to a power of $E=E_{i}$. The details here are left to the reader.

2.1. Strongly minimal sets on elliptic curves. Let $C$ denote a complete nonsingular curve, defined over $k_{0} \subset k$.

Remark 2.17. When $C$ has genus 1 , the sets $\Xi(C, s), s \in \Omega^{1}(C)$, are all nonorthogonal to the constants. Given $s_{1}, \ldots, s_{l} \in \Omega^{1}(C)$, one can ask when there exist $a_{i} \in \Xi\left(C, s_{i}\right)$ with $a_{1}, \ldots, a_{l}$ algebraically dependent over $k$. The answer (a special case of [12], easily retrievable here) is that $s_{1}, \ldots, s_{l}$ should be linearly dependent over the field $L$ of complex multiplication of $C$ (or over $\mathbb{Q}$ if $C$ has no complex multiplication).

By Remark [2.1, and the fact that $\Xi(C, s)$ is non-orthogonal to the constants for $s$ a global 1-form on an elliptic curve $C$ over $k$, the above-mentioned global strictly minimal subsets of higher genus curves are the only order 1 Kolchin-closed subsets of complete nonsingular curves, defined over $k$, with strictly no induced structure. It seems worth noting that on elliptic curves, there do exist strongly minimal sets whose induced structure reduces to a finite set of constants.

Lemma 2.18. Let $E$ be an elliptic curve over $k_{0}$. Let $a_{1}, \ldots, a_{r}$ be distinct points from $E\left(k_{0}\right), r>0$. Assume there are no integers $m_{i}$ (not all 0 ) with $\sum m_{i} a_{i}=0$ (elliptic addition). Also assume the cycle $\sum a_{i}$ has trivial stabilizer, by the translation group of $E$. Let $s$ be a 1-form whose polar cycle is $\sum a_{i}$. 
Then the induced structure on $\Xi(E, s)$ consists only of constants for the points $a_{i}$.

Proof. First we establish the following fact.

Claim. For any $g: E \rightarrow \mathbb{P}^{1}$ (defined over $k$ ) and any rational 1-form $s^{\prime}$ on $\mathbb{P}^{1}$, $s \neq g^{*} s^{\prime}$.

Proof. Suppose $s=g^{*} s^{\prime}$. So $(s)=R+g^{*}\left(s^{\prime}\right)$, where $R$ is the ramification divisor of $g$, effective of degree $\leq d-1,(s)$ is the divisors of poles and zeroes of the 1-form $s$ on $C$, and $g^{*}\left(s^{\prime}\right)$ is the pullback via $g$ of the divisor of zeroes and poles of $s^{\prime}$. (Cf. the proof of Hurwitz' theorem in [5], or Lemma 6.20 in D.Mumford, Algebraic Geometry I: Complex projective varieties, Springer- Verlag, 1995.)

Let $d=\operatorname{deg}(g) . \quad s^{\prime}$ must have a pole at each $b_{i}=g\left(a_{i}\right)$. If $b_{i}$ is a pole of $s^{\prime}$ of order 2 or more, then (counting with multiplicities) $s$ must have at least $2 \operatorname{deg}(g)-\operatorname{deg}(R) \geq d+1$ poles at points $g^{-1}\left(b_{i}\right)$; so at least one of them must be double. Thus since $s$ has no double poles, neither can $s^{\prime}$ have double poles. But any rational 1 -form of $\mathbb{P}^{1}$ has two more poles than zeroes (the canonical divisor has degree -2$)$; so $s^{\prime}$ has at least two poles. Two must be distinct, say $b_{1} \neq$ $b_{2}$. Now $g^{-1}\left(b_{1}\right), g^{-1}\left(b_{2}\right)$ are linearly equivalent cycles on $C$ (with nonnegative integer coefficients; this by definition of linear equivalence). They are formed from two disjoint subsets of points from among $a_{1}, \ldots, a_{r}$. This contradicts the linear independence assumption on the points $a_{i}$.

By 2.10. $\Xi(E, s)$ is orthogonal to the constants, unless $s$ is the pullback by a rational map of a global form on an elliptic curve. But $s$ cannot be such a pullback: any rational map between elliptic curves is a translate of a group homomorphism, and it would follow that $s$ too has no poles, contradicting the assumption.

As in the higher genus case considered above, if $\Xi(E, s)$ has any structure other than constants, then $s$ is the pullback of a rational form on a curve $C^{\prime}$ by a rational map $g: C \rightarrow C^{\prime}$, of degree $>1$. Then $C^{\prime}$ is an elliptic curve or $\mathbb{P}^{1}$. The latter case was already eliminated. In the former case, $g$ is a translate of a non-injective group homomorphism, with finite kernel $K$, and $\sum a_{i}$ must be invariant under translation by $K$, a contradiction.

Thus $\Xi(E, s)$ has no structure except perhaps for constants. These constants must have coordinates from $k$, so their derivative is 0 ; it follows that $s$ has a pole at these points.

Remark 2.19. By contrast, if the polar divisor of $s$ is supported on one point only, then $\Xi(E, s)$ is non-orthogonal to the constants.

2.2. Strictly minimal sets living on $\mathbb{P}^{1}$. There are as many strongly minimal sets on $\mathbb{P}^{1}$ as there are rational functions (since we can write the equation $D x=f(x)$ ); on the other hand, given a strongly minimal set $X$ on $\mathbb{P}^{1}$, there are as many strongly minimal sets on $\mathbb{P}^{1}$ non-orthogonal to it, as there are rational functions. (Namely $g X, g$ a rational or even algebraic function.) Nonetheless there exist families of pairwise orthogonal strictly minimal sets on $\mathbb{P}^{1}$, of arbitrarily large dimension.

Orthogonality to the constants. The following example is due to Tracey McGrail and Dave Marker. It shows 3.1 would be false for the strongly minimal set, $D x=0$. 
Example 2.20. Let $s(x)=\left(c x^{-1}+(x-1)^{-1}\right) d x$. Then $\Xi\left(\mathbb{P}^{1}, s\right)$ is non-orthogonal to $k$ iff $c$ is rational.

The proof uses Theorem 6.12 of [17. In our language, the theorem states:

For $\omega$ a 1-form on $\mathbb{P}^{1}$, defined over $k, \Xi\left(\mathbb{P}^{1}, \omega\right)$ is non-orthogonal to $k$ iff $\omega$ is the pullback of $d x$ or of $c x^{-1} d x$ (constant c) by a rational function $g \in k(X)$.

Note that if we write $\omega=f(x) d x$, the criterion is equivalently stated:

$f$ is the derivative of a rational function, or a constant multiple of the logarithmic derivative of a rational function.

Recall that the residue of a 1-form $\omega$ at a point $p \in \mathbb{P}^{1}$ can be defined as follows. Let $u$ be a local coordinate at $p$. Write $\omega(u)=f(u) d u$. Express $f$ as a Laurent series in $u$. Then the residue is the coefficient of $u^{-1}$.

Note that the logarithmic derivative of a rational function has only simple poles, and has rational integer residues at these poles. (It suffices to check this for $d \log (x-a)$.) Conversely, if $\omega$ is a rational 1-form with this property, let $g$ be a rational function with a zero of order $m$ wherever $\omega$ has a pole with residue $m$. This is possible, since the sum of residues is 0 . Write $(d \log g)=c \omega$ with $c$ a rational function. Then $c$ can have no poles or zeroes, so it must be constant. Comparing residues at some pole of $\omega$, we see that $c=1$. Arguing similarly for the ordinary derivative, we can restate the criterion in a form easy to check in practice:

$\Xi\left(\mathbb{P}^{1}, \omega\right)$ is non-orthogonal to $k$ iff either $\omega$ has no simple poles, or else all poles of $\omega$ are simple, and their residues have rational ratios.

In particular, 2.20 follows.

A test for strict minimality. We would like to give tests for strict minimality and for almost orthogonality of sets living on $\mathbb{P}^{1}$. We will use the following result of Ax; cf. [1] or [17, Lemma 6.10.

Let $k$ be algebraically closed, $K$ an algebraically closed extension of $k$ of transcendence degree 1 . Consider the logarithmic derivative as a map

$$
K^{*} / k^{*} \rightarrow d \log \Omega^{1}(K / k) / d K
$$

Here the domain of the map is the multiplicative group of $K$, modulo the constants. The range is the space of 1 -forms, modulo exact forms.

Lemma 2.21 $(\mathrm{Ax})$. If the group $K^{*} / k^{*}$ is viewed as a $\mathbb{Q}$-space, then the induced map

$$
\left(K^{*} / k^{*}\right) \otimes_{\mathbb{Q}} k \rightarrow \Omega^{1}(K / k) / d K
$$

is injective. In other words, if $f_{1}, \ldots, f_{m} \in K^{*}$ are multiplicatively independent (modulo $\left.k^{*}\right)$, then their logarithmic derivatives are $k$-linearly independent over the exact forms.

Here is an easy-to-apply test for almost-orthogonality among strongly minimal sets living on $\mathbb{P}^{1}$. Recall that the sum of residues of a rational 1-form is 0 ; in particular, a form cannot have a single nonzero residue. 
Lemma 2.22 (rational 1 -forms on $\mathbb{P}^{1}$, defined over the constants). Let $\left\{a_{i}\right\}_{1 \leq i \leq n}$, $\left\{b_{j}\right\}_{1 \leq j \leq n^{\prime}}$ be the distinct simple poles of $\omega, \omega^{\prime}$ respectively, and $c_{i}, e_{j}$ the corresponding residues. Assume $n, n^{\prime}>0$ and that $c_{1}, \ldots, c_{n-1}, e_{1}, \ldots, e_{n^{\prime}-1}$ are linearly independent over $\mathbb{Q}$. Let $X=\Xi\left(\mathbb{P}^{1}, \omega\right), X^{\prime}=\Xi\left(\mathbb{P}^{1}, \omega^{\prime}\right)$. Then $X$ and $X^{\prime}$ are almost orthogonal.

Proof. After a linear fractional change of variable, we may assume $\omega, \omega^{\prime}$ do not have a pole at $\infty$. To motivate, we solve the equations formally for a function $x=\Xi(t)$. Write the equation for $\Xi\left(\mathbb{P}^{1}, \omega\right)$ as

$$
\omega(x) \frac{d x}{d t}=1 .
$$

Inverting, we get

$$
d t=\omega(x) d x
$$

Now write $\omega(x)=r(x)+\sum_{i} c_{i}\left(x-a_{i}\right)^{-1}$, with the $a_{i}$ distinct, $c_{i}$ nonzero, and $r$ a rational function with no simple poles. By integrating the partial fraction expansion of $r$ term by term, we find a rational-function primitive $R$ for $r$; absorbing the constant of integration in $R$, we obtain

$$
t=R(x)+\sum_{i} c_{i} \log \left(x-a_{i}\right) .
$$

Similarly, if $y$ solves $X^{\prime}$, we can write

$$
t=S(y)+\sum_{j} e_{j} \log \left(y-b_{j}\right)
$$

Subtracting the equations, we obtain

$$
\sum c_{i} \log \left(x-a_{i}\right)-\sum e_{j} \log \left(y-b_{j}\right)=S-R .
$$

(We can think of $x, y$ as analytic functions of $t$, defined locally, with essential singularities at $y=b_{j}$ and $x=a_{i}$. Alternatively we can work formally, and directly reach the next equation:)

$$
\sum c_{i} d \log \left(x-a_{i}\right)-\sum e_{j} d \log \left(y-b_{j}\right)=d(S-R) .
$$

Now suppose $X, X^{\prime}$ are not almost orthogonal. Let $x, y$ be algebraically dependent (but non-algebraic) solutions, and $K$ the algebraic closure of $k(x)$ (or of $k(y)$ ). So $S, R$ and hence $S-R$ are in $K$.

By 2.21, it follows that in $\left(K^{*} / k\right) \otimes_{\mathbb{Q}} k$,

$$
\sum_{i}\left(x-a_{i}\right) \otimes c_{i}-\sum_{j}\left(y-b_{j}\right) \otimes e_{j}=0
$$

(with apologies for the mixed additive/multiplicative notation). Now the sum of residues of either form is 0 ; so $c_{n}=-\sum_{i<n} c_{i}, e_{n^{\prime}}=-\sum_{j<n^{\prime}} e_{j}$, and

$$
\sum_{i<n} \frac{x-a_{i}}{x-a_{n}} \otimes c_{i}-\sum_{j<n^{\prime}} \frac{y-b_{j}}{y-b_{n^{\prime}}} \otimes e_{j}=0
$$

By the linear independence assumption, we must have $\left(x-a_{1}\right) /\left(x-a_{n}\right)=1$ in $K^{*} / k$. Solving, we get $x \in k$, a contradiction. 
It would not be difficult to extend 2.22 to a precise criterion.

The appearance of $\mathbb{Q}$ in 2.22 may suggest that almost-orthogonality to $X$ is not definable. The truth of the matter is that every orthogonality class of order 1 strongly minimal sets, other than that of the constants, is definable. This requires different ideas and will be shown elsewhere. (Note however that two strictly minimal Kolchin-closed, irreducible sets living on $\mathbb{P}^{1}$ are not-almost-orthogonal iff one is the image of the other by an element of the definable group $P G L(2, K)$.)

Note that the algebraic points of $\Xi\left(\mathbb{P}^{1}, \omega\right)$ are precisely the poles of $\omega$. Let $X_{*}\left(\mathbb{P}^{1}, \omega\right)$ denote the strongly minimal set obtained by removing these points. Here is the strict minimality test.

Lemma 2.23. Let $\omega$ be a rational 1 -form on $\mathbb{P}^{1}$, defined over $k$. Assume $\omega$ has at least two nonzero residues, and that no two distinct nonzero residues of $\omega$ are rational multiples of each other. Then $X=X_{*}\left(\mathbb{P}^{1}, \omega\right)$ is strictly minimal.

Proof. By the previous remarks, $X$ is orthogonal to $k$. Thus by [7] it is $\aleph_{0^{-}}$ categorical. By [2.9] it suffices to show that $\omega$ is essential. Otherwise, $\omega=g^{*} \omega^{\prime}$ for some $\omega^{\prime}$ and some rational $g: \mathbb{P}^{1} \rightarrow \mathbb{P}^{1}$ of degree $>1$. In particular, the associated sets $X$ and $X^{\prime}$ are not almost orthogonal; indeed, there are solutions $x$ of $X, y$ of $X^{\prime}$ with $y=g(x)$.

Let $a_{i}$ be the poles of $\omega$, with residues $c_{i}$; and $b_{j}$ the poles of $\omega^{\prime}$, with residues $e_{j}$

Now $x, y$ are in $k(x)$. We have $y=g(x)$, so $y-b_{j}$ is a constant multiple of $\prod_{a \in g^{-1}\left(b_{j}\right)}(x-a)^{m_{a}} ; m_{a}$ is the order of the zero of $g-b_{j}$ at $a$.

As $b_{j}$ is a pole of $\omega^{\prime}$, with residue $e_{j}$ say, each $a \in g^{-1}\left(b_{j}\right)$ must be a pole of $\omega$, and by computing locally one finds it has a nonzero residue, namely $m_{a} e_{j}$. If $a=a_{i}$, i.e. $g\left(a_{i}\right)=b_{j}$, write $j=j(i)$.

Claim. $i \mapsto j(i)$ is not $1-1$.

Proof. If for a given $j \in J, g^{-1}\left(b_{j}\right)$ has a single element $a_{i}$, then this element accounts for $d-1$ of the $2 d-2$ ramification points of $g, d=\operatorname{deg}(g)$. This cannot happen at three distinct points. If $i \mapsto j(i)$ is $1-1$, it follows that $\omega^{\prime}$ has at most two nonzero residues, and hence so does $\omega$. But then these residues sum to 0 , so their ratio is -1 , a contradiction.

Thus we can assume $j(1)=j(2)$. So $c_{1} / c_{2}=m_{a_{1}} / m_{a_{2}} \in \mathbb{Q}$. This contradicts the assumption on the irrationality of the residue ratios.

\section{Definability of NON-ORThogonAlity}

Let us call a definable or type-definable set $X$ strictly disintegrated if any $n$ elements of $X$ are independent over the base of definition. (It is known that all order-one sets are non-orthogonal either to $k$ or to a strictly disintegrated set, cf. 7].) Two strictly disintegrated types defined over the same base set $B$ are either orthogonal, or definably isomorphic by a unique definable bijection. (Further, there exists a unique smallest set $B$ over which some element of the non-orthogonality class is defined.) Thus (taking Proposition 1.2 into consideration) the algebraic variety on which $X$ lives is determined up to birational isomorphism by the nonorthogonality class of $X$.

We show here that orthogonality to a strictly disintegrated set $X$ is a definable property of parameters, when $X$ lies on a curve of genus $\geq 1$. 
By contrast, T. McGrail and D. Marker showed by example that orthogonality to the constants does not have this property (2.20).

To avoid tipping the exposition over with technicalities, we assume here some natural facts around the functoriality of the Manin map and the Albanese variety, and return to them in appendices ( $\S 3.3$ and 3.2 below).

Proposition 3.1. Let $C$ be a curve of genus $\geq 1$, or more generally, a subvariety of an Abelian variety. Let $X$ be a strictly disintegrated definable or type-definable subset of $C$. Let $Y_{b}$ be a definable family of irreducible Kolchin-closed definable sets of finite differential order. Then the set of $b$ such that $X$ is orthogonal to the generic type of $Y_{b}$ is a definable set.

Proof. We will take $X$ to be a Kolchin-closed subset of $C$; the proof goes through for the type-definable case with minor linguistic changes.

Fix $b$ and let $Y=Y_{b}$. We will work over the differential field $L=L_{b}$ generated by $b$ (over a field of definition $L_{0}$ for $X, C, Y$ ). We will find conditions equivalent to the orthogonality of $Y$ to $X$. One of these (condition (3) below) will clearly be expressible by a first-order sentence, hence a definable property of $b$.

We may take $Y=Y_{b}$ to be the set of points $v \in V=V_{b}$ at which $D v=s(v)$, where $V_{b}$ is a variety, and $s=s_{b}$ is a certain section of the shifted tangent bundle. This guarantees Zariski-density of $Y$ in $V$. In addition, the algebraic closure of a (generic) element of $Y_{b}$ is the usual field-theoretic algebraic closure, and similarly for definable closure.

Let $d=\operatorname{dim}(V)$. Let $A$ be the Albanese variety of $V$ (cf. §3.2.) We will use the existence - after a possible base change - of a family $A_{b}$ of Abelian varieties and of maps $\iota_{b}: V_{b} \rightarrow A_{b}$, such that $A_{b}$ is an Albanese variety for $V_{b}$. See 3.7 in $\S 3.2$ below.

Let $\mu: A \rightarrow U$ be the Manin map. So $U$ is a vector group, $\mu$ a DCF-definable homomorphism, with kernel of finite Morley rank.

Let $Z=Z_{b}$ be the image of $Y$ under $\mu \circ \iota$.

Also let $B$ be an Abelian variety, into which $C$ is embedded, and let $\mu_{B}: B \rightarrow U^{\prime}$ denote the Manin map. Let $W$ denote the image of $X$ in $U^{\prime}$ under $\mu_{B}$. Let $X_{d}$ be the set of sums of at most $d$ elements of $X$, with respect to the addition in $B$. Note that $X_{d}$ is a set of finite Morley rank; indeed, from the triviality of the geometry of $X$, one deduces immediately that $X_{d}$ has Morley rank $d$. Let $W_{d}$ be the set of sums of at most $d$ elements of $W$, in $U^{\prime}$; it is the image of $X_{d}$ in $U^{\prime}$ under $\mu_{B}$. Using 3.9, let $E=E_{b}$ be a uniformly definable set of affine homomorphisms $U \rightarrow U^{\prime}$, containing the image under $\mu$ of the set $\operatorname{Hom}(A, B)$ of homomorphisms of Abelian varieties $A \rightarrow B$, and closed under composition with the translation group of $U^{\prime}$.

Claim 3.1. The following conditions are equivalent:

1) $X$ is non-orthogonal to the generic type of $Y$.

2) There exists a nonconstant affine homomorphism $h: A \rightarrow B$ of Abelian varieties such that $h(\iota(Y)) \subset X_{d}$.

3) There exists $H \in E$ such that $H(Z) \subset W_{d}$, and $H(Z)$ is infinite.

Proof of Claim. (1) implies (2). Let $a \in Y=Y_{b}$ be a generic point. Let $F=$ $(\operatorname{acl}(a, b) \backslash \operatorname{acl}(b)) \cap X$. By the triviality of the induced structure on $X, F=$ $\left\{c_{1}, \ldots, c_{i}\right\}$ is a finite set; indeed $i \leq d$.

Moreover, using triviality of the algebraic-closure geometry, $i>0$ : by definition of non-orthogonality, there exist independent realizations $a_{1}, \ldots, a_{m}$ of the 
generic type of $Y$, and $c_{1}, \ldots, c_{l}$ of the generic type of $X$, such that the two tuples $a_{1}, \ldots, a_{m}$ and $c_{1}, \ldots, c_{l}$ fork over $b$. Using triviality, some $c_{i}$ forks with $\left(a_{1}, \ldots, a_{m}\right)$. Now if a realization of a trivial regular type forks with an independent set, it must fork with some element of that set (cf. [18]). Thus we may take $m=l=1$.

Let $c=c_{1}+\ldots+c_{i}$, the sum taken in $B$. Then $c \in X_{d}$. Also $c$ is rational over $L(a)$. There exists a rational function $h_{0}$ defined over $L$, with $h_{0}(a)=c$. $h_{0}$ maps $V$ into the Abelian variety $B$, so $h_{0}$ factors as the composition of the Albanese map $\iota: V \rightarrow A$ and an affine homomorphism $h: A \rightarrow B$. We have $h(\iota(a)) \in X_{d}$ for generic $a \in Y$, so $h(\iota Y) \subset X_{d}$. Since the $c_{i}$ are independent elements of $X$ over $b$, it follows that $c \notin \operatorname{acl}(b)$, so $h_{0}$ and hence $h$ are nonconstant.

(2) implies (3). We may assume without loss of generality that $h(0)=0$, i.e., $h$ is a homomorphism. Observe first that $h(\iota Y)$ must be infinite. Otherwise $Y$ is contained in finitely many fibers of $h$. By the Zariski-density of $Y$ in $V$, so is $V$. Being irreducible, $V$ is contained in $h^{-1}(c)$ for some $c$. But since $V$ generates the Albanese $A$ as a ternary group, $A=h^{-1}(c)$. This contradicts the assumption that $h$ is non-constant.

Now $\operatorname{ker}(\mu)$, the Manin kernel, is the smallest definable subgroup of $A$ such that $A / \operatorname{ker}(\mu)$ embeds homomorphically into a vector group (cf. 3.12). Since the composition $\mu_{B} h$ maps $A$ to a vector group, we must have $\left(\mu_{B} h\right)(\operatorname{ker}(\mu))=0$, or $h(\operatorname{ker} \mu)) \subset \operatorname{ker}\left(\mu_{B}\right)$. Thus $h$ induces a definable homomorphism $H: \mu A \rightarrow \mu B$.

We have $H(Z)=H \mu \iota Y=\mu_{B} h \iota Y \subset \mu_{B} X_{d}=W_{d}$.

$X_{d}$ is contained in the algebraic closure of the trivial strongly minimal set $X$, hence is orthogonal to the kernel of the Manin map $\mu_{B}$, so it meets each coset of the Manin map in a finite set. Thus the same is true of $h \iota(Y)$. Since $h \iota(Y)$ is infinite, also $\mu_{B} h \iota(Y)=H(Z)$ is infinite.

(3) implies (1). The strongly minimal set $X$ being trivial, it meets every coset of the Manin kernel in a finite set; hence the map $\mu_{B}$ is finite-to-one on $X$, so $X$ is non-orthogonal to the strongly minimal set $\mu_{B} X=W$. It follows, since $W_{d} \subset \operatorname{acl}(W)$, that every non-algebraic type contained in $W_{d}$ is non-orthogonal to (the generic type of) $X$. In particular, this is true of the generic type of $H(Z)$. Thus a definable image of the generic type of $Y$ is non-orthogonal to $X$, so $Y$ is non-orthogonal to $X$.

Note that in (3), one can quantify over the possible transformations in the uniformly definable set $E$. One can also ("nfcp") uniformly state that a given definable set is infinite, cf. [17, 2.13. Thus (3) is a definable property of $b$. This finishes the proof of the proposition.

Definition 3.2. If the conclusion of Proposition 3.1 holds for a minimal type $p$, we will say that orthogonality to $p$ is definable.

Corollary 3.3. Let $C$ be a variety admitting a non-constant map into an Abelian variety. Let $X$ be a strictly disintegrated definable or type-definable subset of $C$. Assume $X$ is Zariski-dense in $C$. Then orthogonality to $X$ is definable.

Proof. Let $j: C \rightarrow B$ be a non-constant map into an Abelian variety. If $j(X)$ is infinite, then it is also strictly disintegrated (perhaps after removing finitely many points), so Proposition 3.1 applies to $j(X)$; and non-orthogonality to $X$ and to $j(X)$ is the same. If $j(X)$ is finite, then $X$ lies in $C^{\prime}=j^{-1}(p)$ for some $p \in B$; but this contradicts the Zariski-density of $X$ in $C$. 
3.1. Appendix: Irreducibility. A Kolchin-closed set is called irreducible if it is not the union of two proper Kolchin-closed subsets. If one is interested in singular points, irreducibility can be a delicate question. Consider for instance:

Example 3.4. The Kolchin-closed set defined by $2 D x D^{3} x-\left(D^{2} x\right)^{2}-2 x=0$ is reducible.

Indeed, differentiating $2 D x D^{3} x-\left(D^{2} x\right)^{2}-2 x=0$, we obtain $2 D x\left(D^{4} x-1\right)$; so the set decomposes into the solution $x=0$ and $2 D x D^{3} x-\left(D^{2} x\right)^{2}-2 x=0, D^{4} x=1$.

Thus irreducibility of the defining differential polynomial does not imply Kolchinirreducibility.

We do not know whether, in general, singularities included, irreducibility is a definable property in parameters. The question can be reduced to the following: given a point $p=\left(0, p_{2}, \ldots, p_{m}\right)$ and a Kolchin-closed set $X$, known to be irreducible on $x_{1} \neq 0$, is $p$ in the closure of $X$ ?

In all events, for our purposes, there is no harm in discarding a proper Kolchinclosed subset. We can thus restrict attention to Kolchin-closed sets of the form

$$
\Xi(V, s)=\{x \in V: D x=s(x)\}
$$

where $s$ is a regular function on $V$ into the appropriate bundle.

Then clearly any proper Kolchin-closed subset of $\Xi(V, s)$ has the form $\Xi\left(V^{\prime}, s^{\prime}\right)$, where $s^{\prime}=s \mid V^{\prime}$. In this case $\Xi(V, s)$ is irreducible iff the algebraic variety $V$ is irreducible. Definability of the latter is known classically (and proved modeltheoretically in van den Dries' thesis).

(In the one-variable case, the above discussion reads as follows. The Kolchinclosed set is defined by a differential polynomial of order $n, f\left(X, D X, \ldots, D^{n} X\right)$. Consider $f$ as a polynomial in $D^{n} X$, with leading coefficient $h=h\left(X, \ldots, D^{n-1} X\right)$. Let $X^{\prime}$ be defined by $f=0, h \neq 0$. Then $V$ is irreducible iff $f$ is irreducible as a differential polynomial. Since any factors of $f$ would have smaller degree in each variable than $f$, we can quantify over the possible coefficients of such factors. Thus here definability of irreducibility is immediate.)

3.2. Appendix: Families of Abelian varieties. The proof of Proposition 3.1 used a uniformity principle for the construction of the Albanese variety, and of the maximal vector extension of an Abelian variety. The purpose of this subsection is to provide a proof.

The material in this subsection involves no derivations, and we work within algebraic geometry (over an algebraically closed field of characteristic 0). A general reference for this section is [13]. We could not find a direct reference for the uniformity principle we need, though we think it is standard.

By an affine homomorphism between groups we will mean a map of the form $x \rightarrow h(x)+b$, where $h$ is a group homomorphism, and $b$ a constant.

An Albanese variety of a given variety $V$ is an Abelian variety $A$, together with a rational map $f: V \rightarrow A$ such that for any rational map $h$ on $V$ into another Abelian variety $B$, there exists a unique affine homomorphism $g: A \rightarrow B$ with $h=g f$.

Lemma 3.5. Let $V$ be a projective variety, defined over an algebraically closed field $k_{0}, C_{t}$ a generic curve on $V, J_{t}=J\left(C_{t}\right)$ the Jacobian of $C_{t}$. Then the Albanese variety of $V$ can be identified with the quotient of $J_{t}$ by a connected subgroup $S$. 
$S$ is the smallest subgroup of $J_{t}$ such that $J_{t} / S$ is isomorphic to a variety $K$ defined over $k_{0}$.

Proof. $C=C_{t}$ is the intersection of $V$ with a generic linear subspace of codimension $\operatorname{dim}(V)-1$. Let $A$ be the Albanese of $V$. The map $V \rightarrow A$ restricts to $C \rightarrow A$ and hence induces an affine homomorphism $J_{t} \rightarrow A$. The genericity of $t$ guarantees that the image of $C=C_{t}$ in $A$ is not contained in any translate of a proper Abelian subvariety of $A$, so the map $J_{t} \rightarrow A$ is surjective. Clearly it factors through the map $J_{t} \rightarrow K$.

Now we must show that the surjective map $K \rightarrow A$ is an isomorphism. Consider the composed map $C_{t} \rightarrow J_{t} \rightarrow K$. Let $(a, b)$ be a generic point of the graph of this map. Then $a$ is a generic point of $A, a \in C_{t}, b \in K$, and $b$ is rational over $(a, t)$. But the locus of $t$ over $a$ is a rational variety (indexing the linear subspaces of projective space, of appropriate dimensions, passing through $a$ ). Hence, with $a$ fixed, the map $t \rightarrow b$ maps a unirational variety into an Abelian one, and so must be constant. Thus $b$ is rational over $k_{0}(a)$. So there exists a rational map $V \rightarrow K$ such that for generic $t$, the restriction to $C_{t}$ agrees with $C_{t} \rightarrow J_{t} \rightarrow K$. This map induces a morphism $A \rightarrow K$, surjective since $J_{t} \rightarrow K$ is surjective, and since no proper subgroup of $J_{t}$ contains the image of $C_{t}$. It remains only to check that this is an isomorphism, and inverse to the map $K \rightarrow A$. Since $A \rightarrow K$ is surjective, it suffices to check that $A \rightarrow K \rightarrow A=I d_{A}$. Since $J_{t} \rightarrow A$ is surjective, it suffices to check that $J_{t} \rightarrow A \rightarrow K \rightarrow A=J_{t} \rightarrow A$. But $J_{t} \rightarrow A \rightarrow K$ agrees with the previously constructed $J_{t} \rightarrow K$, since they agree on $C_{t}$. Thus it suffices to check that $J_{t} \rightarrow K \rightarrow A=J_{t} \rightarrow A$. This is clear by definition of the map $K \rightarrow A$.

Lemma 3.6. Let $C_{b}$ be a uniformly definable family of smooth nonsingular curves, indexed by a variety $B$. Let $J_{b}$, $j_{b}$ be a uniformly definable family of varieties and maps $j_{b}: C_{b} \times C_{b} \rightarrow J_{b}$. Assume that for generic $b^{*} \in B, J_{b^{*}}$ is the Jacobian of $C_{b^{*}}$, and $j_{b^{*}}(x, y)=x-y$. Then the same is true for all $b$ on a Zariski-open subset of $B$.

Proof. The following facts hold true for $b=b^{*}$ (with respect to some auxiliary formulas and integers), and can be witnessed by a first-order formula in $b$, hence are true for almost all $b \in B$ :

(a) $J_{b}$ is a group variety, and a projective variety.

(b) $j_{b}(x, z)=j_{b}(x, y)+j_{b}(y, z)$

(c) For any fixed $p \in C_{b}$, any element of $J_{b}$ is the sum of $d$ elements of $j_{b}\left(p, C_{b}\right)$.

(d) If $x_{1}+\ldots+x_{d}=y_{1}+\ldots+y_{d}$ for points $x_{1}, \ldots, y_{d} \in j_{b}\left(p, C_{b}\right)$, then the cycle $x_{1}+\ldots+x_{d}-y_{1}-\ldots-y_{d}$ is linearly equivalent to 0 on $C_{b}$.

(In property (d), the cycle is the cycle of zeroes and poles of a rational function on $C_{b}$. Since such a function exists for any $x_{1}, \ldots, x_{d}, y_{1}, \ldots, y_{d}$ satisfying the hypothesis, it must by compactness exist within a uniformly definable family; so a first order formula true of $b^{*}$ can ascertain the truth of the fact.)

Conversely to (d), since by (a) $J_{b}$ is an Abelian variety, if the cycle $x_{1}+\ldots+$ $x_{d}-y_{1}-\ldots-y_{d}$ is linearly equivalent to 0 , then $x_{1}+\ldots+x_{d}=y_{1}+\ldots+y_{d}$ in $J_{b}$.

It follows from (a)-(d), using Abel's criterion, that $J_{b}$ is the Jacobian of $C_{b}$.

Lemma 3.7. Let $V_{b}$ be a uniformly definable family of projective varieties, indexed by a variety $B$. Let $A_{b}$ be the Albanese of $V_{b}$. Then the family $A_{b}$ is uniformly definable. 
Proof. Say $B, V$ are defined over an algebraically closed field $k_{0}$. Let $C_{b, t}$ be a generic curve on $V_{b}$, i.e. the intersection of $V_{b}$ with a linear subspace of projective space of codimension $\operatorname{dim}\left(V_{b}\right)-1$, chosen generically over $k_{0}(b)$. Let $j_{b, t}:\left(C_{b, t}\right)^{2} \rightarrow$ $J_{b, t}$ be the Jacobian of $C_{b, t}$. The family $J_{b, t}, j_{b, t}$ is uniformly definable by 3.6 .

By 3.5 for generic $b^{*}$ and $t$, the Albanese $A_{b^{*}}$ of $V_{b^{*}}$ is isomorphic via an isomorphism $h_{b^{*}, t}$ to $J_{b^{*}, t} / S_{b^{*}, t}$, where $S_{b^{*}, t}$ is a connected subgroup. Moreover, $S_{b^{*}, t}$ has no $k_{0}\left(b^{*}\right)$-isotrivial factors, i.e. no nonzero homomorphisms into Abelian varieties defined over finite extensions of $k_{0}\left(b^{*}\right)$. (To see this we may apply 3.5 over $k_{0}\left(b^{*}\right)^{\text {alg }}$.)

For almost all $b$ and $t, S_{b, t}$ is a connected subgroup of $J_{b}, A_{b}$ is an Abelian variety defined over $k_{0}(b)$, and $A_{b}=J_{b, t} / S_{b, t}$. Moreover, for almost all $b$, for generic $t, S_{b, t}$ has no $k_{0}(b)$-isotrivial factors. The deduction of this last fact from the corresponding fact at $b^{*}$ is explained in [6]. (The point there is that the variety $W_{b^{*}}$ over $k\left(b^{*}\right)$, whose function field is the finite extension of the function field of $S_{b^{*}, t}$ generated by the $l$-torsion points of $S_{b^{*}, t}$, remains irreducible when $b^{*}$ is specialized to $b$, for almost all $b$.)

Hence by 3.5 for almost all $b, A_{b}$ is the Albanese of $V_{b}$.

(An alternative argument: the Albanese $A_{b}$ is isomorphic to $J_{b, t} / \bar{S}_{b, t}$ for some $\bar{S}_{b, t}$. It remains to argue that $\bar{S}_{b, t}=S_{b, t}$. Clearly $\bar{S}_{b, t} \subset S_{b, t}$. Since $S_{b, t}$ is connected, if $\bar{S}_{b, t}$ is a proper subgroup, then $\operatorname{dim}\left(A_{b}\right)>\operatorname{dim}\left(A_{b^{*}}\right)$. However this dimension can be viewed as the dimension of a cohomology group, and by Grothendieck's semi-continuity must be constant on some Zariski-open subset of $B$. )

The proof of Proposition 3.1 will require a uniform family $M_{b}$ of commutative algebraic group varieties, extensions of $A_{b}$ by vector groups, such that the prolongation map $T_{D} M_{b} \rightarrow M_{b}$ splits. This splitting property can trivially be captured by a first-order formula in the language of differential fields, and this suffices for the purposes of [3.1. Still it is nice to know that one can take $M_{b}$ to be an algebraic family.

If $A$ is an algebraic group, a vector extension is an algebraic group $B$ together with an exact sequence $0 \rightarrow V \rightarrow B \rightarrow A \rightarrow 0$, with $V$ a vector group. According to [22], the dimension of such a group $B$ is bounded. It follows that there exists a unique one $0 \rightarrow V_{A} \rightarrow A^{\prime} \rightarrow A \rightarrow 0$ enjoying the universal property: for any $0 \rightarrow V \rightarrow B \rightarrow A \rightarrow 0$, there exists a unique morphism $A^{\prime} \rightarrow B$ compatible with $I d: A \rightarrow A$ and with some morphism $V_{A} \rightarrow V$. This $A^{\prime}$ is called the maximal vector extension of $A$. It is obviously functorial in $A$.

Lemma 3.8. Let $A_{b}$ be a uniformly definable family of Abelian varieties, indexed by a variety $B$. Let $M_{b}=A_{b}{ }^{\prime}$ be the maximal extension of $A_{b}$ by a vector group. Then the family $M_{b}$ is uniformly definable.

Proof. A proof similar to that of 3.7 can be given, using the following two facts.

1) By [22] or [25], extensions of an Abelian variety $A$ by $G_{a}$ are classified by $H^{1}\left(A, \mathcal{O}_{A}\right)$. Given an extension $0 \rightarrow G_{a} \rightarrow B \rightarrow A_{b} \rightarrow 0$ of $A_{b}$ by $G_{a}$, the corresponding classifying element of $H^{1}\left(A_{b}, G_{a}\right)$ can be found uniformly, as the proofs there show.

2) By [5], III, 12.8, 12.9, the $H^{1}\left(A, \mathcal{O}_{A}\right)$ form a uniformly definable family. (Using the notation there, let $f: X \rightarrow Y$ be a projective morphism. Let $\mathcal{F}$ be the structure sheaf of $X$. We want to show that the family $H^{i}\left(X_{y}, \mathcal{F}_{y}\right)$ is uniformly definable. By generic flatness, we can decompose $Y$ into locally closed sets $Y_{j}$ such 
that $f: f^{-1} Y_{j} \rightarrow Y_{j}$ is flat. So we may assume $f$ is flat, i.e., $\mathcal{F}$ is flat over $Y$. By $12.8, \operatorname{dim}_{k(y)} H^{i}\left(X_{y}, \mathcal{F}_{y}\right)$ is upper semicontinuous. Thus there exists a partition of $Y$ into locally closed sets $Y_{i}$, with $h_{i}$ constant on $Y_{i}$. By 12.9 , there exists a vector bundle $E_{i}$ on $Y_{i}\left(E_{i}=\operatorname{Spec} R^{i} f_{*}(\mathcal{F})\right.$ on $\left.Y_{i}\right)$ such that $H^{i}\left(X_{y}, \mathcal{F}_{y}\right)$ is isomorphic to the fiber of $E_{i}$ at $y$.)

Alternatively, one can use the bounds on degrees of projective embeddings of vector extensions of Abelian varieties, found in [2].

3.3. Appendix: Image of the Manin functor. This appendix contains results that we believe appear in [3], implicitly or explicitly, but we could not conveniently quote.

Let $\Psi A$ denote the image of an Abelian variety $A$ under the Manin map. Given two Abelian varieties $A$ and $B, \Psi$ induces a homomorphism from $\operatorname{Hom}(A, B)$ into the group of definable homomorphisms from $\Psi A \rightarrow \Psi B . \operatorname{End}(A)=\operatorname{Hom}(A, A)$ is never uniformly definable: it is countable. Nor is the group $\operatorname{Hom}_{\text {def }}(\Psi A, \Psi B)$ of DCF-definable homomorphisms uniformly definable. Even for the 1-dimensional vector space $V$ over a differentially closed field $K, \operatorname{Hom}_{\text {def }}(V, V)$ is the ring generated by $K$ and the derivation $D$; it is not contained in any uniformly definable family. Nevertheless, we show that the image of $\operatorname{Hom}(A, B)$ in $\operatorname{Hom}(\Psi A, \Psi B)$ is contained in a uniformly definable subgroup (subring if $A=B$ ).

Lemma 3.9. Let $A$ and $B$ be Abelian varieties. Then the image of $\operatorname{Hom}(A, B)$ in $\operatorname{Hom}(\Psi A, \Psi B)$ is contained in a uniformly definable family. Moreover if $A, B$ vary in a uniformly definable family, then the image of $\operatorname{Hom}(A, B)$ is contained in a uniformly definable family of homomorphisms $\Psi A \rightarrow \Psi B$, varying uniformly with the parameter.

Towards the proof of 3.9, we recall the following functorial construction of the Manin maps. Given an Abelian variety $A$, let $A^{\prime}$ be the maximal extension of $A$ by a vector group (Appendix 3.2). $A \rightarrow A^{\prime}$ is a functor on the category of algebraic groups.

On the other hand, given a differential field $L$, the prolongation functor takes algebraic groups $B$ over $L$ to exact sequences $0 \rightarrow T_{e} B \rightarrow B_{D} \rightarrow \pi_{B} B \rightarrow 0$ of algebraic groups over $L$. Here $T_{e} B$ is the tangent space to $B$ at the identity element $e$.

If $K$ is a differential field extension of $L$, the derivation of $L$ induces a map $i_{D}: B \rightarrow B_{D}$ (given, in coordinates, by $\left(a_{1}, \ldots, a_{n}\right) \mapsto\left(a_{1}, \ldots, a_{n}, D a_{1}, \ldots, D a_{n}\right)$ ).

We write $A^{*}$ for $A^{\prime}{ }_{D}, \Psi A^{\prime}$ for $T_{e} A^{\prime}$. Since $A^{*}$ is a vector group extension of $A^{\prime}$, the universal property of $A^{\prime}$ yields an algebraic group homomorphism $f_{A^{\prime}}$ : $A^{\prime} \rightarrow A^{*}$. Thus $0 \rightarrow \Psi_{A^{\prime}} \rightarrow A^{*} \rightarrow A^{\prime} \rightarrow 0$ is split; we obtain a projection $1-f_{A^{\prime}} \pi_{A^{\prime}}: A^{*} \rightarrow \Psi_{A^{\prime}}$.

The Manin map $\mu_{A^{\prime}}$ of $A^{\prime}$ is then defined to be the composition

$$
A^{\prime} \rightarrow i_{D} A^{\prime *} \rightarrow 1-f_{A^{\prime}} \pi_{A^{\prime}} \Psi A^{\prime} .
$$

For any homomorphism $h: A \rightarrow B$, the functoriality of $A \rightarrow A^{\prime}$ and of $B \rightarrow B_{D}$ yields a map $\Psi(h): \Psi A^{\prime} \rightarrow \Psi B^{\prime}$. This is a morphism of algebraic groups. Since $\Psi A^{\prime}$ and $\Psi B^{\prime}$ are vector groups, it is a linear transformation.

Proof of 3.9 (Written for the case $A=B$ ). To obtain the Manin map on $A$ itself, we let $\Psi A=\left(\Psi A^{\prime}\right) / \mu_{A^{\prime}}\left(V_{A}\right)$, where $V_{A}$ is the kernel of $A^{\prime} \rightarrow A$. Then $\mu_{A^{\prime}}$ induces a 
natural map $\mu_{A}: A \rightarrow \Psi A$. An endomorphism $g$ of $\Psi A^{\prime}$ respecting $\mu_{A}\left(V_{A}\right)$ induces an endomorphism of $\Psi A$, denoted $\pi_{*}(g)$.

Any endomorphism $h$ of $A$ yields functorially an endomorphism $h^{\prime}$ of $A^{\prime}$. By construction, the image of $h^{\prime}$ in $\Psi A^{\prime}$ is a linear transformation. Thus the image of $\operatorname{End}(A)$ in $\operatorname{End}(\Psi A)$ is $\left\{\pi_{*}(g): g \in G L\left(A^{\prime}\right)\right\}$. Since $G L\left(A^{\prime}\right)$ is uniformly definable, so is the image of $\operatorname{End}(A)$.

Lemma 3.10. $\mu_{A^{\prime}}: A^{\prime} \rightarrow \Psi A^{\prime}$ is a surjective map of $A^{\prime}$ into the algebraic vector group $\Psi A^{\prime}$.

For any morphism $j: B \rightarrow A$ of algebraic groups over $L, j_{*} \Psi B^{\prime}=\mu_{A^{\prime}}\left(j^{\prime} B^{\prime}\right)$. In particular, $\mu_{A^{\prime}}\left(j^{\prime} B^{\prime}\right)$ is an algebraic subgroup of $\Psi A^{\prime}$.

Proof. Note that the kernel of $\mu_{A^{\prime}}$ has finite Morley rank. Thus the image of $\mu_{A^{\prime}}$ has Morley rank $\omega \operatorname{dim}\left(A^{\prime}\right)$. It is a definable subgroup of $\Psi A^{\prime}$, and we have $\operatorname{dim}\left(\Psi A^{\prime}\right)=$ $\operatorname{dim}\left(A^{\prime}\right)$ as algebraic groups, so the Morley rank of $\Psi A^{\prime}$ is also $\omega \operatorname{dim}\left(A^{\prime}\right)$. Therefore $\mu_{A^{\prime}}$ is surjective.

The definition of the map $j_{*}: \Psi B^{\prime} \rightarrow \Psi A^{\prime}$ and the verification of the functoriality $j_{*} \mu_{B^{\prime}}=\mu_{A^{\prime}} j^{\prime}$ are left to the reader. Given this, $\mu_{A^{\prime}}\left(j^{\prime} B^{\prime}\right)=j_{*} \mu_{B^{\prime}}\left(B^{\prime}\right)=j_{*} \Psi B^{\prime}$.

Remark 3.11. As pointed out to us by Thomas Scanlon, the image of the endomorphism ring is simply contained in the space of linear transformations $\Psi A \rightarrow \Psi A$. While the map $\mu: A^{\prime} \rightarrow \Psi\left(A^{\prime}\right)$ depends on the differential algebraic structure, the image of $V_{A}$ under this map is an algebraic group, and hence (say by quantifier elimination for DCF0) depends only on the given derivation on the base field. Thus the functor $\Psi(A)=\Psi\left(A^{\prime}\right) / \Psi\left(V_{A}\right)$ can be defined on the category of algebraic varieties over the base field $L$. In particular, the images of algebraic homomorphisms are again algebraic homomorphisms.

Remark 3.12. Any differentially definable homomorphism $A \rightarrow V$, where $V$ is a vector group, factors through the Manin map $\mu$. The Manin kernel $\operatorname{ker}(\mu)$ is the smallest definable subgroup $S$ of $A$ such that $A / S$ embeds into a vector group.

Proof. The second statement is equivalent to the first, and the first follows from the same statement for $A^{\prime}$, the maximal vector extension of $A$. Let $f$ be a differential rational group homomorphism on $A^{\prime}$ into a vector group. On the Manin kernel $\operatorname{ker}(\mu), f$ coincides with a rational map $F$ (since the derivative of $a \in \operatorname{ker}(\mu)$ is rational in a.) Taking Zariski closure, $F$ gives a rational group homomorphism $A^{\prime} \rightarrow V$. This group homomorphism must be trivial by definition of the maximal vector extension (otherwise $A^{\prime}$ splits into the kernel of $F$, and a vector group). So $f$ vanishes on $\operatorname{ker}(\mu)$, as required.

\section{Differential FIELDS ADMITTING QUANTIFIER ELIMINATION}

\section{Is every superstable differential field differentially closed?}

In this section we answer the question by constructing theories of differential fields that admit quantifier elimination, but are not differentially closed. Such fields are automatically $\omega$-stable.

This confirms a prediction made in [19]. By the work there, any superstable differential field has enough points in definable sets non-orthogonal to nontrivial types. Thus one must look at types with trivial geometry to settle the problem. 
Our main new tool here is the definability of non-orthogonality, i.e., Propositions 2.1 and 3.1

Applying this, we define a theory $T$ in the language of differential fields, $L=$ $\{+,-, \cdot, 0,1, D\}$. Recall,

Proposition 2.1. Let $C$ be a curve of genus $\geq 1$, or more generally, a subvariety of an Abelian variety. Let $X$ be a strongly minimal subset of $C$; assume the induced structure on $X$ is trivial. Let $Y_{b}$ be a definable family of Kolchin-closed definable sets of finite differential order. Then the set of $b$ such that $X$ is orthogonal to the generic type of $Y_{b}$ is definable.

By Proposition 2.1 or Lemma 2.13, strictly minimal sets $X$ living on curves $C$ as in 2.1 do exist; moreover, we may find $C$ and $X$ defined over $\mathbb{Q}$. Fix such $C, X$. Suppose $X$ is defined by a formula $\varphi(v)$.

Definition of $T=T(X)$. (1) The universal part $T_{\forall}$ of $T$ consists of the following (i), (ii), and ( $\left.\mathrm{ii}^{\prime}\right)$.

(i) the theory of differential fields of characteristic 0 (DF0)

(ii) $X$ has no solution, i.e., $\neg(\exists x)(\varphi(x))$,

(ii') $X$ has no solution even in the algebraic closure.

This is actually an axiom scheme. Let $h$ be a monic polynomial over $F$, in one variable. Note that if $h(x)=0$ (in the algebraic closure $\widetilde{F}$ of $F)$, then $D x=-h^{*}(x) / h^{\prime}(x)$, where $h^{*}$ is the result of applying $D$ to the coefficients of $h$. Thus differential algebraic statements about solutions to $h$ reduce to algebraic ones. There exists therefore a sentence $\xi_{h}$ over $F$ stating that, among tuples of roots of $h$ in the algebraic closure of $F$, there are no solutions to $X$. One can initially take it to be the negation of an existential sentence in the language of fields, and then, using quantifier elimination in $\mathrm{ACF}$, translate to a quantifier-free formula. The axioms (ii') ensure that $\xi_{h}$ holds for all such $h$.

(2) The rest of $T$ consists of the following (iii) and (iv).

(iii) The theory of algebraically closed fields of characteristic 0 (ACF0),

(iv) This axiom scheme will have an axiom for each instance of the following data:

$U, W$ differential-algebraic varieties over $\mathbb{Q}, \pi: U \rightarrow W$, such that $U_{b}:=$ $\pi^{-1}(b)$ has finite differential order, for each $b \in W$. We take $U$ to be of the form considered in $\S 3.1$, by the discussion there,

$$
W_{1}=\left\{b: U_{b} \text { is irreducible }\right\}
$$

is a definable set in DCF0; we implicitly consider it to be defined by a quantifier-free formula.

Let $p_{b}$ denote the generic type of $U_{b}$ (for $b \in W_{1}$ ).

By Proposition 3.1,

$$
W_{2}:=\left\{b \in W_{1}: p_{b} \text { is orthogonal to } X\right\}
$$

is also definable; say by $\theta(w)$, a quantifier-free formula.

Finally, we impose the following axiom: $(\forall w \in W)(\theta(w) \rightarrow(\exists u)(u \in U$, $\pi(u)=w))$.

Remark 4.1. By definition, the axiom group (1)(ii') is the set of universal consequences of (1)(i),(ii) and (2)(iii). One can write it down explicitly, but this is not 
necessary for our purposes. We will merely use the fact that every model of (1) extends to a model of (1) + (2)(iii).

Lemma 4.2. The theory $T=T(X)$ is consistent. In fact, $T_{\forall}$ is consistent, and any model of $T_{\forall}$ can be extended into a model of $T$.

Proof. Consistency of $T_{\forall}: \mathbb{Q}^{\text {alg }}$, with the trivial derivation, is a model of $(1)(\mathrm{i}, \mathrm{ii})$ and (2)(iii), hence of (1)(ii') as well. This uses the fact that $X$ has no algebraic solutions. That in turn is part of the assumption of trivial induced structure on $X$.

We now show that any model $M$ of $T_{\forall}$ can be extended into a model of $T$. We can view $M$ as embedded in a universal domain $\mathbb{U}$ for DCF0.

First extend $M$ to the algebraic closure $M^{\text {alg }}$. By Theorem 7 in Chapter X, section 7 in [14], we can extend the derivation from $M$ to $M^{\text {alg }}$ as well. The truth of (1)(ii') in $M$ guarantees the truth of (1) in $M^{\text {alg }}$. Thus we assume that our model $M$ is algebraically closed.

To extend $M$ into a model of $T$ we need to iterate the following process. Suppose we are given $U, \pi, W$ as in (iv) and $b \in W(M)$ such that $\theta(b)$ holds.

To make (iv) hold, we simply add a generic solution $c \in U_{b}$ (taken from $\mathbb{U}$ ). But we must show that after adding the generic $c$ to $M$, (ii) and (ii') remain true. This guarantees that in the end we get a model of $T$. Thus let $N=M(c)^{\text {alg }} \subset \mathbb{U}$. We must show that (1) continues to hold for $N$. Since $N$ is algebraically closed, it suffices to show (1)(ii), i.e.

Claim. $M(c)^{\text {alg }} \cap X=\emptyset$.

Proof of Claim. We work in DCF0, treating $M$ as a substructure of $\mathbb{U}$. "acl" and orthogonality refer to $\mathbb{U}$.

Suppose $e \in M(c)^{\operatorname{alg}} \cap X$. Since $M \models T_{\forall}, e \notin M$. Thus $e \in \operatorname{acl}(M(c)) \backslash \operatorname{acl}(M)$. So $c, e$ fork over $M$. But $c$ realizes $p_{b}$ over $M$. This contradicts the assumption that $\theta(b)$ holds, so that $p_{b}$ is orthogonal to $X$.

This completes the proof of the claim, and of the lemma.

Lemma 4.3. Let $M$ be a model of $T(X)$. Let $E$ be a Kolchin-closed set defined over $M$. If some generic type of $E$ is orthogonal to $X$, then $E(M) \neq \emptyset$.

Proof. We use induction on the Morley rank of $E$ (computed in DCF0).

Note first that for any $n$, in some elementary extension of $M$, there exists a generic point of $L(n)=\left\{x: D^{n} x=0\right\}$. This is because the generic type of $L(n)$ has finite differential order, is internal to the constants, and is orthogonal to $X$.

By compactness, in some elementary extension $N$ of $M$, there exists also a point $a$ differentially transcendental over $M$.

We may assume $E$ is irreducible. If $E$ has finite Morley rank, then an instance of axiom scheme (2)(iv) of $T(X)$ explicitly states that $E$ has a point. Otherwise, let $e=\left(e_{1}, \ldots, e_{k}\right)$ be a generic point of $E$ over $M$. Then some $e_{i}$, say $e_{1}$, is differentially transcendental over $M$. Let

$$
E^{\prime}=\left\{\left(x_{2}, \ldots, x_{k}\right):\left(a, x_{2}, \ldots, x_{k}\right) \in E\right\} .
$$

Then $E^{\prime}$ has smaller Morley rank than $E$. Moreover, some generic type of $E^{\prime}$ is orthogonal to $X$. Otherwise, there would exist $b \in E^{\prime}$, generic over $M(a)$, with $\operatorname{acl}(M(a, b)) \cap X \neq \emptyset$; but $(a, b)$ is a generic point of $E$, contradicting the assumption on $E$. Thus, by induction, there exists $b^{\prime} \in E^{\prime}(N)$. So $\left(a, b^{\prime}\right) \in E(N)$. Since $M$ is an elementary submodel of $N, E(M) \neq \emptyset$. 
Proposition 4.1. $T$ is a model completion of $T_{\forall}$.

Proof. To show that $T$ is a model completion of $T_{\forall}$, we prove the following two facts:

(a) If $M$ is an existentially closed model of $T_{\forall}$, then $M$ is a model of $T$.

(b) If $M \models T, A$ is a substructure of $M$, and $\phi$ is a quantifier-free formula over $A$, with a solution in some $N \models T, A \subset N$, then $\phi$ has a solution in $M$.

For (a) it suffices to show that $M$ satisfies (iv), i.e., $\theta(b) \rightarrow U_{b} \neq \emptyset$. Assume $M \models \theta(b)$. By Lemmas 4.2 and 4.3 , $M$ has an extension $M^{\prime} \models T_{\forall}$ in which $U_{\bar{b}} \neq \emptyset$. Since $M$ is existentially closed, the same is true in $M$. So $M$ is a model of $T$.

Now let $M, N, A, \phi$ be as in (b). Let $c \in N, N \models \phi(c)$. The usual trick allows us to assume $\phi$ is positive. (If $\phi$ contains a negative part $f(c) \neq 0$, replace $c$ by $c d$, where $d=f(c)^{-1}$, and replace that negative part by $f(c) d=1$.) Let $E_{0}$ be the smallest Kolchin-closed set, defined over $A$, such that $c \in E_{0}$. In $N, \operatorname{tp}(c / A)$ is a generic type of $E_{0}$, and $\operatorname{acl}(A c) \cap X \subset N \cap X=\emptyset$. It follows that some generic type of $E_{0}$ is orthogonal to $X$ (in DCF0). In $M$, let $E$ be a component of $E_{0}$ whose generic type is orthogonal to $X$. By $4.3 . E(M) \neq \emptyset$. Let $c^{\prime} \in E(M)$. Then $\phi\left(c^{\prime}\right)$.

Remark 4.4. It follows that $T$ admits quantifier elimination, since any model completion of a universal theory admits quantifier elimination (cf. Theorem 13.2 in [24]), or directly from (b). Note also that any quantifier eliminable theory of differential fields is $\omega$-stable (cf. Lemma 10.2 in [17]).

$T$ is complete, as it admits quantifier elimination, and $\mathbb{Q}$ is a substructure of any model of $T$.

Remark 4.5. T admits elimination of imaginaries. This follows from the following lemma, applied with $T_{2}=T_{\forall}, T_{1}=$ theory of differential fields of characteristic 0 .

Lemma 4.6. Let $T_{1} \subset T_{2}$ be universal theories in the same language, with model completions $\tilde{T}_{1}, \tilde{T}_{2}$. Assume that, in $\tilde{T}_{1}$, every 0 -definable function is equivalent to the interpretation of a function symbol. (Thus, if $M_{i} \models T_{i}$ and $M_{2}$ is a substructure of $M_{1}$, then $M_{2}$ is closed under the 0-definable functions in $M_{1}$ ).

(1) If $\tilde{T}_{1}$ is ( $\kappa$-)stable, so is $\tilde{T}_{2}$.

(2) If $\tilde{T}_{1}$ admits coding of finite sets, so does $\tilde{T}_{2}$.

(3) If $\tilde{T}_{1}$ is stable and admits weak elimination of imaginaries, so does $\tilde{T}_{2}$.

(4) If $\tilde{T}_{1}$ is stable and admits elimination of imaginaries, so does $\tilde{T}_{2}$.

Proof. The stability statement (1) is immediate, by counting types; it suffices to count quantifier-free types; any model of $T_{2}$ embeds as a substructure of a model of $T_{1}$, and any consistent $T_{2}$-quantifier-free type is also consistent with $T_{1}$. Thus the number of types over a model of $\tilde{T}_{2}$ of size $\kappa$ is at most the number of types over a model of $\tilde{T}_{1}$ of the same size.

Let us now prove (2). Let $U_{2} \subset U_{1}$ be saturated models of $\tilde{T}_{2}$ and $\tilde{T}_{1}$, respectively. Let $F$ be a finite set of elements of $U_{2}$. Since $\tilde{T}_{1}$ admit coding of finite sets, this set is coded by some tuple $b$ from $U_{1} ; F=D(b)$, where $D(y)$ is a $\tilde{T}_{1}$-uniformly definable family of distinct finite sets of fixed size $m$. Since $b$ is definable from the elements of $F$, it follows that $b \in U_{2}$. We have $D(b)^{U_{2}}=D(b) \cap U_{2}=F$. If $b^{\prime}$ is another element of $U_{2}$, with the same type as $b$, then it also has the same type as 
an element of $U_{1}$. So $D\left(b^{\prime}\right)$ is an $m$-element set, not equal to $D(b)$. Whether or not all the elements of $D\left(b^{\prime}\right)$ are in $U_{2}$, we cannot have $D(b)=D\left(b^{\prime}\right)$. This shows that $b$ codes $F$ in $U_{2}$ too.

To prove (3), suppose $e$ is an imaginary element in a model $M_{2}$ of $\tilde{T}_{2}$; let $V$ denote the union of the (non-imaginary) sorts of the language. Let $E=\operatorname{acl}_{\tilde{T}_{2}}(e) \cap V$. We shall show that $e$ is definable over $E$.

We have $e=f(c)$, for some definable function $f$ and some tuple $c$ of standard elements of $M_{2}$. Let $c^{\prime} \in U_{2}$ realize $\operatorname{tp}\left(c / \operatorname{acl}_{\tilde{T}_{2}}(e)\right)$, with $c^{\prime} T_{2}$-independent from $M_{2}$ over $e$.

Let $p=\operatorname{stp}_{T_{2}}\left(c^{\prime} / c, E\right)$.

Embed $M_{2}$ in a model $M_{1}$ of $T_{1}$; choose $M_{1}$ independent from $c^{\prime}$ over $M_{2}$ (in the sense of $\left.T_{1}\right)$.

Let $\varphi(x, y)$ be a quantifier-free formula over $E$, and let $\theta(x)$ be the $p(x)$-definition of $\varphi$, in the sense of $T_{1}$. Then $\theta$ is almost over $M_{2}$. Since $\tilde{T}_{1}$ eliminates imaginaries, $\theta$ is coded in the standard sorts $V$. More precisely, there exist an element $\epsilon$ of $V$ and a finite set $F_{\epsilon}$ of tuples of $V$ defined in a quantifier-free way from $\epsilon$, with $\epsilon$ serving as canonical parameter, such that $\theta$ is quantifier-free definable from any element of $F_{\epsilon}$.

$\theta$ is an extendible definition (i.e. $\left\{\varphi(x, d) \Leftrightarrow \theta(d): d \in U_{1}\right\}$ is consistent). We have indeed

$$
\varphi\left(c^{\prime}, d\right) \Leftrightarrow \theta(d)
$$

for all $d \in M_{1}$.

This remains true when restricted to $\tilde{T}_{2}$ : the quantifier-free formula $\theta$ is almost over $M_{2}$, i.e., it has finitely many conjugates under $\operatorname{Aut}\left(U_{1} / M_{2}\right)$; so it certainly has finitely many conjugates in $\operatorname{Aut}\left(U_{2} / M_{2}\right)$. Since $M_{2}$ is a model, it is over $M_{2}$. Moreover, $\varphi\left(c^{\prime}, e\right) \Leftrightarrow \theta(e)$ for $e \in M_{2}$. Thus $\theta$ is also the $\operatorname{tp}\left(c^{\prime} / M_{2}\right)$-definition of $\varphi$, in the sense of $\tilde{T}_{2}$.

By independence of $c^{\prime}, M$ over $e, \theta$ is over $\operatorname{acl}_{T_{2}}(e)$. But it is coded in $V$. So $\theta$ is over $E$.

In particular, taking for $\varphi$ the formula $f(x)=f(y)$, we see that $e$ is definable over $E$. Weak elimination of imaginaries follows.

(4) is immediate from (2) and (3).

Remark. (4) is not valid without the stability assumption: take $T_{1}$ to be the empty theory in a language with a single binary relation $R$, and $T_{2}$ the theory stating that $R$ is an equivalence relation.

Remark 4.7. There exists a continuum of different substructure complete theories of differential fields of characteristic 0 .

Sketch of proof. Using Proposition 2.1, choose a strictly minimal set $X_{n}$ living on a curve $C_{n}$ of genus $n$, defined over $\mathbb{Q}$. For any set of natural numbers $S$, consider the class of differential fields $M$ of characteristic 0 such that, for $n \in S, X_{n}$ has no points in the algebraic closure of $M$. This is a universal theory; one shows it has a model completion. The axioms of the model completion state that any Zariski-dense Kolchin-closed subset $Y$ of a variety $V$ has a point, provided that $Y$ is orthogonal to each $X_{n}, n \in S$. This does not immediately appear to be firstorder as such. However, let $d=d(V)$ be the dimension of the Albanese variety $A$ of $V$. We saw in $\S 2$ that as $V$ runs in a definable family of varieties, $d(V)$ remains 
bounded. We also saw that if $Y$ and $X_{n}$ are non-orthogonal, then there exists a dominant rational map $V \rightarrow X$, hence a surjective rational map from $A$ to the Jacobian of $C_{n}$. So $d(V) \geq n$. Thus the proviso in the axioms can be restated:

Any Zariski-dense Kolchin-closed subset $Y$ of a variety $V$ has a point, provided that $Y$ is orthogonal to each $X_{n}, n \in S, n \leq d(V)$.

This last statement is seen to be first-order in the same way as in the proof of 3.1 .

Remark 4.8. Any superstable theory in a countable language can be interpreted in a superstable differential field.

Sketch of proof. Consider for convenience a superstable theory $T$ in a language $L$ with a single predicate $R$ of arity $r$. It is convenient to define the theory of superstable fields in a language $\{+, \cdot, D, R\}$; however, $R$ will be 0 -definable from the other relations, so at the cost of complicating the theory it can be removed from the language.

Pick a strictly minimal set $Y$ on the affine line; it need not live on the affine line, so it could be the image of a set produced by Proposition 2.1 or it could be one of Rosenlicht's or Shelah's sets. Pick also a curve $E$ over $\mathbb{Q}$ of genus $\gamma>2 r$. Choose $E$ whose Jacobian is not isogenous to a power of an elliptic curve. (For example, take a generic linear section of a product of $r$ non-isogenous elliptic curves.) Pick an $r$-dimensional affine subspace $\bar{S}$ of $\Omega^{1}(E)$, defined over $\mathbb{Q}^{\text {alg }}$, such that $g \bar{S} \cap \bar{S}=\emptyset$ for $g \in G=\operatorname{Aut}(E / k)$ (Since $G$ is finite, this will be the case for almost all $r$ dimensional affine subspaces.) Let $S: \mathbb{A}^{r} \rightarrow \bar{S}$ be an isomorphism of affine spaces. Then:

The theory $T *$ will state that the field is an algebraically closed differential field.

$R$ is an $r$-ary relation on $Y\left(R \subset Y^{r}\right)$.

The induced structure $(Y, R)$ is a model of $T$.

The strongly minimal sets $\Xi(E, S(t))$ do not have a point if $t \in R$. However, any Kolchin-closed set orthogonal to all these $\Xi(E, S(t))$ does have a point.

Further details are left to the reader.

\section{REFERENCES}

[1] Ax, James, On Schanuel's Conjectures, Annals of Math. 293 (1971), pp. 252-268. MR 43:3215

[2] Buium, Alexandru, Effective bound for the geometric Lang conjecture. Duke Math. J. 71 (1993), no. 2, pp. 475-499. MR 95c:14055

[3] A. Buium, Differential Algebraic Groups of Finite Dimension, Lecture Notes in Mathematics 1506, Springer-Verlag 1992. MR 93i:12010

[4] G. Cherlin, S. Shelah, Superstable fields and groups, Annals of Math. Logic 18 (1980), pp. 227-270. MR 82c:03045

[5] Robin Hartshorne, Algebraic Geometry, Springer-Verlag 1977. MR 57:3116

[6] E. Hrushovski, Proof of Manin's thorem by reduction to positive characteristic, in Model Theory and Algebraic geometry, E. Bouscaren ed., Lecture Notes in Mathematics 1696, Springer-Verlag 1998, pp. 197-205 MR 2000c:11202

[7] E. Hrushovski, ODE's of order 1 and a generalization of a theorem of Jouanolou (to appear).

[8] E. Hrushovski, Almost orthogonal regular types, Annals of Pure and Applied Logic 45. 139155. 1989. MR 91k:03083

[9] E. Hrushovski, Ž. Sokolovic, Strongly minimal sets in differentially closed fields, to appear in Transactions of the AMS

[10] E. L. Ince, Ordinary differential equations, Dover, New York 1944. MR 6:65f

[11] E. R. Kolchin, Constrained extensions of differential fields, Advances in Math. vol 12, 1974, pp. 141-170. MR 49:4982 
[12] E. R. Kolchin: Algebraic Groups and Algebraic Dependence, American Journal of Mathematics, 90 1968, pp. 1151-1164. MR 39:1460

[13] S. Lang, Abelian varieties, Springer-Verlag 1983. MR 84g:14041

[14] S. Lang, Algebra, Addison-Wesley, 1965. MR 33:5416

[15] S. Lang, Introduction to algebraic geometry, Addison - Wesley, Reading 1972. MR 49:8983

[16] A. Macintyre, On $\omega_{1}$-categorical theories of fields, Fund. Math. 71 (1971) pp. 1-25 MR 45:48

[17] D. Marker, Model theory of differential fields, in: Model theory of fields, D. Marker, M. Messmer, A. Pillay, Lecture Notes in Logic 5, Springer-Verlag, Berlin-Tokyo 1996. MR 2001j:12005

[18] Anand Pillay, Geometric Stability Theory, Oxford Logic Guides No 32, 1996. MR 98a:03049

[19] A. Pillay., Differential Galois theory II, Annals of Pure and Applied Logic 88, 1997, 181-191 MR 99m:12010

[20] B. Poizat, Groupes Stables, Nur Al-Mantiq Wal-Ma'rifah, Paris, 1987. MR 89b:03056

[21] B. Poizat, Une Théorie de Galois imaginaire, Journal of Symbolic Logic 48 1983, 1151-1170. MR 85e:03083

[22] M. Rosenlicht, Extensions of vector groups by Abelian varieties, American Journal of Mathematics 80 (1958) pp. 685-714. MR 20:5780

[23] M. Rosenlicht, The nonminimality of the differential closure, Pacific J. Math vol. 52 No. 2, 1974 pp. 529-537. MR 50:4556

[24] G. Sacks, Saturated model theory, Benjamin, 1972. MR 53:2668

[25] J.-P. Serre, Groupes algébriques et corps de classes, Hermann, 1975. MR 57:6032

[26] S. Shelah, Uniqueness and characterization of prime models over sets for totally transcendental first order theories, J. Symbolic Logic 37 (1972) 107-113. MR 47:4787

[27] S. Shelah, Differentially closed fields, Israel Journal of Mathematics v. 16, 1973, pp. 314-328. MR 49:8856

[28] P. Samuel, Complément à un article de Hans Grauert sur la conjecture de Mordell, Publ. Math. IHES 29 (1966), pp. 54-62. MR 34:4272

Department of Mathematics, Hebrew University, Jerusalem, Israel

E-mail address: ehud@sunset.ma.huji.ac.il

Department of Mathematical Sciences, Tokai University, Hiratsuka 259-1292, Japan

E-mail address: itai@ss.u-tokai.ac.jp 